\title{
MicroRNA Profiling Reveals an Abundant ssc- miRNA-148a-3p that Promotes Proliferation and Differentiation during Intramuscular Adipogenesis in Chinese Guizhou Congjiang Pigs Breeds by targeting PPARGC1A
}

Lulin Tan

Guizhou University https://orcid.org/0000-0003-1185-3570

Zhaojun Chen

Guizhou Academy of Agricultural Sciences

Mingde Teng

Guizhou University

Bin Chen

Guizhou University

Houqiang Xu ( $\nabla$ gzdxxhq@163.com )

Guizhou University https://orcid.org/0000-0002-4696-5671

\section{Research}

Keywords: miRNA, Pig, Intramuscular Preadipocyte differentiation, RNA-seq, miR-148a-3p

Posted Date: August 19th, 2021

DOl: https://doi.org/10.21203/rs.3.rs-818015/v1

License: (a) This work is licensed under a Creative Commons Attribution 4.0 International License.

Read Full License 


\section{Abstract}

\section{Background}

Intramuscular fat development is regulated by a series of complicated processes, and non-coding RNA (ncRNA) such as microRNA (miRNA) plays a critical role during intramuscular preadipocyte proliferation and differentiation development in pigs. In present research, we detected the expression profiles of miRNA during different differentiation stages, namely, day 0 (D0), day 4 (D4), and day 8 (D8), of intramuscular preadipocytes from the longissimus dorsi muscle of Chinese Guizhou Congjiang pigs to provide first insights into their potential involvement in intramuscular preadipocyte development. And we investigated the function of miR-148a-3p in adipocyte proliferation, apoptosis, and differentiation.

Results

A total of 67,95 , and 16 differentially expressed (DE) miRNAs were detected between D4 and D0, between D8 and D0, and between D8 and D4, respectively. We further characterized the role of miR-148a-3p which was differentially expressed and highest expressed abundance in D0, D4, and D8. To explore the role of miR-148a-3p in porcine intramuscular preadipocyte, miR-148a-3p mimics and inhibitors were used to perform miR-148a-3p overexpression and knockdown, respectively. Overexpression of miRNA-148a-3p increased the number of intramuscular preadipocytes in the S/G2 phase of the cell cycle and decreased the proportion of cells in the $\mathrm{G} 0 / \mathrm{G} 1$ phase. Moreover, it promoted proliferation by regulation of $c y c l i n B$, cyclin $G 1$, cyclin D1, CDK2, CDK3, and $C D K 4$ and inhibited apoptosis of intramuscular preadipocyte by regulating the expression of Caspase-3, Bax, and Bcl-2. Meanwhile, the mimics of miR-148a-3p dramatically promoted intramuscular preadipocyte differentiation and upregulated the expression levels of adipogenic marker genes PPARY, FASN, FABP4, HSL, APOE, LPL, and CEBPa. Furthermore, miR-148a-3p promoted intramuscular preadipocyte differentiation via restraining the AMPK/ACC/CPT1C signaling pathway. PPARGC1A was identified as a target gene of miR-148a-3p by luciferase activity and western blotting assays.

\section{Conclusion}

Our study provides novel insights into the regulatory mechanisms underlying intramuscular preadipocyte development and identified amount of miRNAs whose regulatory potential will need to be explored in the future. Our results establish that miR-148a-3p promoted adipocyte differentiation by targeting PPARGC1A.

\section{Background}

Intramuscular fat (IMF) content plays a key role in various quality traits of meat such as flavor, tenderness, and juiciness [1]. The proven technique of isolation and in vitro culture of primary intramuscular preadipocytes make it possible for us to understand the mechanisms of IMF deposition [2]. Intramuscular fat content is positively correlated with the number of fat-cell and the size of fat-cell [3]. 
Therefore, understanding the proliferation and differentiation of pig intramuscular preadipocytes may provide valuable information in animal body development and improvement of meat quality [4].

Over the last decade, genome-wide analyses of mammalian transcriptomes discovered that more than $50 \%$ of transcripts are not translated into proteins but act as transcriptional noise or functional RNAs, including non-coding RNA (ncRNA) [5]. Generally, ncRNAs are divided into small or short ncRNA and long ncRNAs (IncRNAs) [6]. MicroRNAs (miRNA) are a kind of 22 nt small non-coding RNAs that direct posttranscriptional repression of mRNA targets in mammals [6]. In recent years, a large number of miRNAs have been identified in eukaryotic organisms from nematodes to humans [7-9]. Moreover, miRNAs have been regarded as key regulators because they play a critical role in various biological processes, such as animal development, cell proliferation, and differentiation, transcriptional regulation, and homeostasis [10]. Numerous studies have demonstrated that miRNAs regulate intramuscular preadipocytes adipogenesis and differentiation through versatile gene-regulatory mechanisms [11]. Wei et al. [12] have researched the expression pattern of miRNAs with longissimus dorsi muscles from the higher and lower longissimus dorsi content of pigs by RNA-Seq and found 73 differentially expressed miRNAs were potential candidates influencing the longissimus dorsi trait by their target genes. Several researchers have reported that miR-199a-3p [13], miR-21 [14], miRNA-143a-3p [15], miR425 [16] influenced the fat deposition by their target genes which involved in cell proliferation and differentiation. However, there is limited research on the expression profiles and the function of miRNAs in intramuscular preadipocytes during different differentiation stages, especially in livestock intramuscular preadipocytes differentiation; for example, in pigs.

Chinese Guizhou Congjiang pigs, known as the fragrant and tender of its meat, have excellent meat qualities. This study aimed to identify miRNAs with potential roles in Chinese Guizhou Congjiang pigs' intramuscular preadipocyte proliferation and differentiation. In the present study, the expression characters of miRNAs were detected using RNA sequencing (RNA-Seq) during different differentiation stages (day 0 (D0), day 4 (D4), day 8 (D8) of intramuscular preadipocyte differentiation in Chinese Guizhou Congjiang pigs. We further characterize on abundant and differentially expressed miRNA, miR148a-3p, which functions by target of PPARGC1A and inhibits intramuscular preadipocyte proliferation and promote intramuscular preadipocyte differentiation. Our research will extensively benefit the improvement of pigs breeding in China and provide new insight, describing the genetic mechanism of the excellent meat quality of Chinese Guizhou Congjiang pigs.

\section{Materials And Methods}

\section{Assurance of regulatory compliance and animals}

Three 3-day-old Chinese Guizhou Congjiang piglets were obtained from local livestock farms. All experimental procedures were approved by the Guizhou University Animal Care and Use Committee, Guizhou, China.

\section{Intramuscular preadipocyte culture and differentiation}


Piglets were killed by venous administration of sodium pentobarbital ( $30 \mathrm{mg} / \mathrm{kg}$ of body weight) and sterilized by $75 \%$ ethanol to obtain samples of longissimus doris muscle. Then, the tissue was washed with phosphate-buffered saline (PBS) by three times and cut into $1-2 \mathrm{~cm}$ pieces. Immediately, the tissue was digested with $2 \mathrm{mg} / \mathrm{mL}$ collagenase type $\$ at $37^{\circ} \mathrm{C}$ for $65 \mathrm{~min}$ and shakes well every $10 \mathrm{~min}$. The digested tissue was terminated with equal volume DMEM/F12 growth medium and filtrated with gauze for removing the undigested tissues. The filtrated solution was then filtered with a 200 and $400 \mu \mathrm{m}$ cell strainer and centrifuged at $1500 \mathrm{r} / \mathrm{min}$ for $10 \mathrm{~min}$ to collect the progenitor cells. The cells were cultured in DMEM/F12 growth medium containing $10 \%$ fetal bovine serum (FBS) and $1 \%$ penicillin-streptomycin at $37^{\circ} \mathrm{C}$ with an atmosphere of $5 \% \mathrm{CO}_{2}$. Because preadipocytes attach much earlier than myoblasts, the cultured cells were washed with PBS three times for removing unadherent cells and insoluble myofibrillar proteins after culturing for 2 hours [4]. After the preadipocytes reach confluence (D0), the growth medium was changed with induction medium which is the growth medium supplement with $5 \mathrm{mM} \mathrm{IBMX}, 1 \mu \mathrm{M}$ DEX, and $5 \mu \mathrm{g} / \mathrm{mL}$ insulin. After 2 days, the cells were subjected to maintenance medium (growth medium supplemented with $5 \mu \mathrm{g} / \mathrm{mL}$ insulin) for an additional 2 days (D4) and then changed every 2 days until day 8 (D8). Three samples were collected at day $0,4,8$, respectively for sequencing.

\section{RNA extraction, library preparation, clustering, and sequencing}

Total RNA was isolated at each time point (D0, D4, and D8) using the Trizol reagent (Takara, Dalian, China). RNA integrity was assessed using the RNA Nano 6000 Assay Kit of the Bioanalyzer 2100 system (Agilent Technologies, CA, USA) and 1\% agarose gels electrophoresis, which showed that the RNA integrity number (RIN) values of all samples were larger than 9.8. RNA concentration was measured using Qubit@ RNA Assay Kit in Qubit® 2.0 Fluorometer (Life Technologies, CA, USA). A total amount of $3 \mu \mathrm{g}$ total RNA per sample was used as input material for the small RNA library. Sequencing libraries were generated using NEBNext® Multiples Small RNA Library Prep Set for Illumina ${ }^{\circledR}$ (NEB, USA.) following manufacturer's recommendations and index codes were added to attribute sequences to each sample. Library quality was assessed on the Agilent Bioanalyzer 2100 system using DNA High Sensitivity Chips. The clustering of the index-coded samples was performed on a cBot Cluster Generation System using TruSeq PE Cluster Kit v3-cBot-HS (Illumia) according to the manufacturer's instructions. After cluster generation, the library preparations were sequenced on an Illumina Hiseq 2500/2000 platform and 50bp single-end reads were generated.

\section{Data analysis}

The data were analyzed through the following principle: Firstly, raw data (raw reads) of fastq format were processed through custom perl and python scripts. Secondly, low quality reads, including reads containing ploy-N, with 5'adapter contaminants, without 3'adapter or the insert tag, containing ploy $\mathrm{A}$ or $\mathrm{T}$ or $\mathrm{G}$ or $\mathrm{C}$, were removed from raw data, and Q20, Q30, and GC-content of the raw data were calculated. Thirdly, the remaining clean reads were mapped to the porcine reference genome sequence (Sus scrofa 11.1) by Bowtie [17] without mismatch. The known porcine miRNA were searched using modified 
software mirdeep2 [18] and srna-tools-cli. Fourthly, the hairpin structures of miRNA precursor were used to predict novel miRNA through the software miREvo [19] and mirdeep2 [18].

\section{Differentially expressed (DE) miRNAs and pathway analysis}

The miRNA expression levels were estimated by TPM (transcript per million) method. The differential expression of miRNAs were performed using the DESeq R package (1.8.3) [20]. The corrected $P$ value $\leq 0.05$ was set as the threshold for significantly differential expression by default. The function of DE miRNAs was predicted by the GO analysis of their target mRNAs. Gene Ontology (GO) enrichment analysis was performed for DE miRNAs using GOseq [21]. We used KOBAS [22] software to test the statistical enrichment of the target gene in KEGG (Kyoto Encyclopedia of Genes and Genomes) pathway enrichment.

\section{Construction of the miRNA-mRNA Network}

According to the miRanda and RNAhybrid, the DE mRNA-miRNA interaction pairs were obtained. The network was performed using Cytoscape [23]. The function analyses of DE mRNAs in the ceRNA network were performed by KEGG pathway analysis [22].

\section{cDNA synthesis and quantitative real-time RT-PCR}

The RNA reversed to cDNA with RevertAid First Strand cDNA Synthesis Kit (Thermo, Waltham, USA). The stem-loop primers were used for reverse-transcribed cDNA of miRNA (Table S1). Meanwhile, U6 (for miRNA) and GAPDH were used as the reference gene for the normalization of the data. The gene expression levels were calculated using the $2^{\square \Delta \triangle \mathrm{Ct}}$ method and qPCR primers were listed in Table S2.

\section{Cells Transfection}

Mimics (Sequence: 5囚-UCAGUGCACUACAGAACUUUGU-3囚, 5囚-AAAGUUCUGUAGUGCACUGAUU-3囚 ), Mimics NC (Sequence: 5囚-UUCUCCGAACGUGUCACGUTT-3囚, 5囚-ACGUGACACGUUCGGAGAATT-3囚), inhibitor (Sequence: 5囚-ACAAAGUUCUGUAGUGCACUGA-3区), and inhibitor NC (Sequence: 5囚CAGUACUUUUGUGUAGUACAA-3区) oligonucleotides of miRNA-148a-3p were synthesized by GenePharma (Shanghai, China). For proliferation, intramuscular preadipocyte was transfected with the miR-148a-3p mimics (60 nM), mimics NC (60 nM), inhibitors (60 nM), and inhibitor NC (60 nM) using the FuGENE®HD Transfection Reagent (Promega Corporation, Madison, USA) according to the manufacturer's introduction when cell confluence reached $40 \%$ for proliferation. For apoptosis and differentiation, when cell confluence reached $80 \%$, miR-148a-3p mimics, mimics NC, inhibitors, and inhibitor NC were transfected into cells in the same way.

\section{Cell Proliferation Assay}

Porcine intramuscular preadipocytes were plated in 96-well culture plates. Each treatment had eight independent replicates. Cell proliferation was measured at $0,24,48,72,96,120,144,168,192 \mathrm{~h}$ after 
translated with miR-148a-3p mimics, mimics NC, inhibitor, inhibitor NC according to the manufacturer's protocol using cell counting kit-8 (CCK-8) assay (APExBIO, Houston, USA). Furthermore, EdU was used to assess cell proliferation following the manufacturer's instructions (APExBIO, Houston, USA).

\section{Flow Cytometry for Cell Cycle assays}

To analysis the effect of miR-148a-3p in different periods of the cell cycle, we analyzed the cell cycle with miR-148a-3p mimics, mimics NC, inhibitor, inhibitor NC groups using the cell cycle testing kit (BD, New Jersey, USA). The cells cultivated in six-well plates were collected and then centrifuged at $800 \mathrm{~g} / \mathrm{min}$ for $5 \mathrm{~min}$. The supernatant was discarded, and the cells were washed with PBS and fixed with $75 \%$ alcohol for 24 hours. The fixed cells were centrifuged at $1000 \mathrm{~g} / \mathrm{min}$ for $10 \mathrm{~min}$, washed with PBS to remove alcohol, stained with $\mathrm{PI} / \mathrm{RNase}$ for 15 minutes in the dark at room temperature, and detected for flow cytometry (FACS Canto II plus, BD BioSciences, USA).

\section{Flow Cytometry for Apoptosis Assays}

To analysis the effects of miR-148a-3p in cell apoptosis by Annexin V-FITC/PI staining assay (BD, New Jersey, USA). After transfection, cells from the different treatment groups (three independent replicates per treatment) were washed three times with PBS buffer $(\mathrm{pH}=7.4)$, collected by trypsinization, washed again with PBS, and then resuspended in $1 \mathrm{ml} 1 \times$ binding buffer (BD, New Jersey, USA). Afterward, we incubated the cells for $15 \mathrm{~min}$ in the dark at room temperature in the presence of Annexin V-FITC $(5 \mu \mathrm{l})$

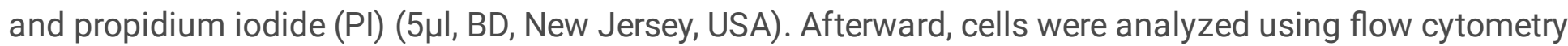
(FACS Canto II plus, BD BioSciences, USA).

\section{Hoechst 33342 and PI Dual Staining Assays}

Hoechst 33342 and PI double staining (Solarbio, Beijing, China) were performed to analyze cell apoptosis. In brief, after transfection with miR-148a-3p for 48 hours, cells were washed with PBS for three times and incubated with Hoechst 33342 and PI for 30 min at $4^{\circ} \mathrm{C}$. Then the cells were washed with PBS and the fluorescence signal was assessed using a fluorescence microscope (Nikon 80i, Tokyo, Japan).

\section{Luciferase Activity Assay}

The wild-type and mutant-type $3^{\prime} U T R$ of PPARGC1A were cloned into a pmir-GLO vector which was made by a manufacturer (TsingKe Biotech, Chongqing, China). The miR-148a-3p mimics, mimics NC, PmirGLO3'UTR-WT, PmirGLO-3'UTR-MUT were co-transfected into 293T cells, when the cell confluence reached about $80 \%$. After transfection $24 \mathrm{~h}$, the cell was washed with PBS and lysed with $500 \mu \mathrm{L}$ passive lysis buffer (PLB). The dual-luciferase activity was measured with the Dual-Luciferase ${ }^{\circledR}$ Reporter Assay System (Promega Corporation, Madison, USA) according to the manufacturer's instructions.

\section{Oil Red 0 Staining, Triglyceride, and Cholesterol analysis}


The cultured cells were washed thrice with PBS, fixed in 4\% paraformaldehyde for 30 min $₫$ and stained with Oil Red $O$ for $20 \mathrm{~min}$. The stained cells were washed three times with PBS and token photos to observe under an inverted microscope (Nikon, Tokyo, Japan).

\section{Western Blotting}

We extracted protein from different treatment porcine intramuscular preadipocytes using RIPA Lysis Buffer (Solarbio, Beijing, China), according to the manufacturer's instructions. We prepared total protein and detected protein concentrations using the Bradford method. Proteins were then separated by SDSpolyacrylamide gel electrophoresis (SDS-PAGE) and subsequently transferred to a PVDF membrane and blocked with $5 \%$ skim milk powder solution for $2 \mathrm{~h}$ at $37^{\circ} \mathrm{C}$ temperature. We incubated membranes overnight with the primary antibodies, washed the membranes with TBST three times, 8 minutes each time, and incubated them for $2 \mathrm{~h}$ with horseradish peroxidase-conjugated secondary antibodies (Abcam, Cambridge, England). We purchased anti-PPARY and anti-CEBPa from Abcam (Abcam, Cambridge, England). Anti-CDK4, anti-Phospho- $A M P K$, anti- $A M P K$, anti- $A C C$ and anti- $L P L$ were purchased from Thermo fisher scientific (Thermo, Waltham, USA). Anti-cyclinD1 was purchased from Aviva Systems Biology (Aviva Systems Biology, California, USA). Anti-Phospho-ACC was purchased from Biorbyt Company (Biorbyt, Cambridge, England). Anti-GAPDH, anti-PCNA, anti-PPARGC1A, and anti-CPT1C were purchased from Proteintech Group (Proteintech, Wuhan, China). Protein bands were detected after treatment with BeyoECL Moon (Beyotime, Shanghai, China).

\section{Statistical analysis}

Data were analyzed by one-way ANOVA using the procedures of SPSS Version 18.0 statistical software. Differences were considered significant at $P<0.05$.

\section{Results}

\section{Characters of RNA-seq results and quality control}

In our present study, we constructed nine small RNA libraries (d0_1, d0_2, d0_3, d4_1, d4_2, d4_3, d8_1, d8_2, d8_3) using porcine intramuscular preadipocyte with different stages of differentiation. A total of $16050754,20667858,18521184,15356301,14585607,16028024,14393598,15354839$, and 13928901 high quality clean reads were obtained from d0_1, d0_2, d0_3,d4_1, d4_2, d4_3, d8_1, d8_2 and d8_3 respectively (Table S3). Additionally, the clean reads were mapped to the pig reference genome (Sus scrofa 11.1), and the mapping ratio ranged from $98.71 \%$ to $99.10 \%$ (Table S4). These results indicated that our data were satisfied for further analysis. The size distribution of mappable reads was similar in nine libraries, and the majority of reads were distributed to $23 \mathrm{nt}$ in length, accounting for $52.87 \%$ (d0_1, Figure S1 a), 49.57\% (d0_2, Figure S1 b) , 45.09\% (d0_3, Figure S1 c), 51.65\% (d4_1, Figure S1 d), 49.74\% (d4_2, Figure S1 e), 52.70\% (d4_3, Figure S1 f), 43.12\% (d8_1, Figure S1 g), 46.74\% (d8_2, Figure S1 h), and $41.36 \%$ (d8_3, Figure $\mathrm{S} 1 \mathrm{i}$ ). The secondary mappable reads were distributed to $22 \mathrm{nt}$ in length, 
ranging from $20.54 \%$ to $28.29 \%$. The detailed length distribution of clean reads was displayed in Table S5.

\section{Identification of porcine known and novel candidate miRNAs}

The mapped small RNA was used to identify the known mature miRNAs and precursors. MiRBase 20.0 database was used as a reference to obtain the known miRNA. A total of 326, 327, 321, 321, 318, 323, 319,319 , and 325 porcine known miRNAs were detected from d0_1, d0_2, d0_3, d4_1, d4_2, d4_3, d8_1, d8_2 and d8_3 groups, respectively (Table 1). Furthermore, we performed base bias analysis for known miRNAs; the results indicated that $\mathrm{U}$ is the most preferred base at the first nucleotide in $20 \mathrm{nt}, 21 \mathrm{nt}, 22 \mathrm{nt}$, $23 \mathrm{nt}$ and $24 \mathrm{nt}$ lengths of known miRNAs (Figure S2). Meanwhile, miRNA nucleotide bias at each position was displayed in Figure S3.

Table 1. Summary of known miRNA.

\begin{tabular}{cllll} 
Types & Mapped mature & Mapped hairpin & Mapped uniq sRNA & Mapped total sRNA \\
\hline Total & 349 & 314 & 3,2813 & $119,781,230$ \\
\hline d0_1 & 326 & 297 & 3,619 & $13,426,884$ \\
\hline d0_2 & 327 & 299 & 3,916 & $17,276,190$ \\
\hline d0_3 & 321 & 291 & 3,974 & $14,393,249$ \\
\hline d4_1 & 321 & 291 & 3,573 & $12,929,026$ \\
\hline d4_2 & 318 & 292 & 3,336 & $12,410,722$ \\
\hline d4_3 & 323 & 294 & 3,532 & $13,359,639$ \\
\hline d8_1 & 319 & 289 & 3,655 & $11,596,430$ \\
\hline d8_2 & 319 & 295 & 3,486 & $13,014,289$ \\
\hline d8_3 & 325 & 300 & 3,722 & $11,374,801$
\end{tabular}

The numbers and percentage of the reads mapping to certain known classes of RNA sequences, including rRNA, tRNA, snRNA, snoRNA, exon, and intron were shown in Table S6. The certain known classes of RNA sequences were excluded, and the remaining reads were analyzed to identify the potential novel miRNAs. A total of 76, 79, 76, 69, 67, 71, 55, 59, 61 novel potential miRNAs were predicted from d0_1, d0_2, d0_3, d4_1, d4_2, d4_3, d8_1, d8_2 and d8_3 groups, respectively (Table 2). The base bias analysis for novel predicted miRNAs were performed. The length of predicted novel miRNA was $22 \mathrm{nt}$, while the length of known miRNA was mainly clustered in $23 \mathrm{nt}$. The first nucleotide bias of the novel predicted $22 \mathrm{nt}$ miRNA is $\mathrm{G}$ difference with $\mathrm{U}$ in known miRNA at $22 \mathrm{nt}$. The first nucleotide bias both of known and novel predicted $23 \mathrm{nt}$ and $24 \mathrm{nt}$ is $\mathrm{U}$ (Figure S4). The predicted novel miRNA nucleotide bias at each position was displayed in Figure S5. 
Table 2. Summary of predicted novel miRNA

\begin{tabular}{|c|c|c|c|c|c|}
\hline Types & $\begin{array}{l}\text { Mapped } \\
\text { mature }\end{array}$ & $\begin{array}{l}\text { Mapped } \\
\text { star }\end{array}$ & $\begin{array}{l}\text { Mapped } \\
\text { hairpin }\end{array}$ & $\begin{array}{l}\text { Mapped uniq } \\
\text { SRNA }\end{array}$ & $\begin{array}{l}\text { Mapped total } \\
\text { sRNA }\end{array}$ \\
\hline d0_1 & 76 & 20 & 84 & 182 & 5,762 \\
\hline d0_2 & 79 & 19 & 87 & 194 & 7,486 \\
\hline d0_3 & 76 & 16 & 82 & 184 & 6,927 \\
\hline d4__1 & 69 & 10 & 74 & 150 & 3,926 \\
\hline d4__2 & 67 & 13 & 70 & 146 & 3,201 \\
\hline d4_3 & 71 & 12 & 78 & 155 & 3,745 \\
\hline d8_1 & 55 & 15 & 65 & 141 & 4,024 \\
\hline d8_2 & 59 & 13 & 64 & 140 & 4,109 \\
\hline d8_3 & 61 & 13 & 67 & 138 & 4,306 \\
\hline
\end{tabular}

\section{Differentially expressed (DE) of miRNA during intramuscular preadipocyte differentiation}

The TPM (tag per million) was used to calculate and normalize the relative expression level of miRNA. A total of 471 miRNA was obtained from three differentiation stages in Table S7. The TPM density distributions of the miRNA expression level in different groups were similar (Figure S6). Three comparison groups (D4 vs D0, D8 vs D0, D8 vs D4) were set to analyze the DE miRNAs during the intramuscular preadipocyte differentiation. A total of $67,95,16$ miRNAs were detected differentially expressed in group D4 vs D0 and D4 vs D0, D4 vs D0 and D8 vs D4, D8 vs D0, and D8 vs D4, respectively (Table 3). The twenty most up-regulated in group D4 vs D0 and D8 vs D0 are presented in Tables 4 and 5. The Venn diagram of DE miRNAs at different time-point comparisons was shown in Figure 1. We found 7 miRNAs that were commonly differentially expressed genes during the entire differentiation process (Figure 1D). The volcano plot of differentially expressed miRNAs was shown in Figures 2A, B, and C. To further explore the potential function of miRNA, we prepared a clustered heatmap (Figure 3).

Table 3. The number of DE miRNAs in the D4 vs. D0, D8 vs. D0, and D8 vs. D4 comparison.

\begin{tabular}{llll} 
Groups & DE miRNAs & Upregulated miRNAs & Downregulated miRNAs \\
\hline D4 vs D0 & 67 & 37 & 30 \\
\hline D8 vs D0 & 95 & 48 & 47 \\
\hline D8 vs D4 & 16 & 8 & 8
\end{tabular}

Table 4. The top 20 most up-regulated miRNAs at the D4 compared to the D0. 


\begin{tabular}{|c|c|c|c|c|c|}
\hline miRNA & $\begin{array}{l}\text { D4 } \\
\text { (readcount) }\end{array}$ & $\begin{array}{l}\text { D0 } \\
\text { (readcount) }\end{array}$ & log2FoldChange & pval & padj \\
\hline $\begin{array}{l}\text { ssc-miR-708- } \\
5 p\end{array}$ & 5,220 & 982 & 2.41 & $4.22 \mathrm{E}-52$ & $1.15 \mathrm{E}-49$ \\
\hline $\begin{array}{l}\text { ssc-miR-708- } \\
3 p\end{array}$ & 2,165 & 462 & 2.23 & $8.91 \mathrm{E}-40$ & $1.22 \mathrm{E}-37$ \\
\hline ssc-miR-146b & 1,843 & 192 & 3.26 & $3.10 \mathrm{E}-32$ & $2.82 \mathrm{E}-30$ \\
\hline ssc-miR-181a & 29,864 & 9,898 & 1.59 & $1.89 \mathrm{E}-25$ & $1.29 \mathrm{E}-23$ \\
\hline $\begin{array}{l}\text { ssc-miR-455- } \\
3 p\end{array}$ & 916 & 370 & 1.31 & $2.42 \mathrm{E}-14$ & 1.32E-12 \\
\hline $\begin{array}{l}\text { ssc-miR-126- } \\
3 p\end{array}$ & 2,570 & 1,002 & 1.36 & $5.40 \mathrm{E}-14$ & $2.46 \mathrm{E}-12$ \\
\hline ssc-miR-181b & 6,461 & 2,787 & 1.21 & 2.07E-13 & 7.06E-12 \\
\hline $\begin{array}{l}\text { ssc-miR-455- } \\
5 p\end{array}$ & 6,890 & 2,899 & 1.25 & $4.11 \mathrm{E}-13$ & $1.25 \mathrm{E}-11$ \\
\hline ssc-miR-378 & 11,981 & 6,153 & 0.96 & 1.74E-09 & 4.76E-08 \\
\hline $\begin{array}{l}\text { ssc-miR-199b- } \\
5 p\end{array}$ & 41,476 & 21,059 & 0.98 & 8.29E-08 & 2.06E-06 \\
\hline ssc-miR-19b & 4,578 & 2,143 & 1.10 & $3.12 \mathrm{E}-07$ & 7.10E-06 \\
\hline $\begin{array}{l}\text { ssc-miR-148a- } \\
3 p\end{array}$ & 382,600 & 204,421 & 0.90 & 4.94E-07 & $9.64 \mathrm{E}-06$ \\
\hline $\begin{array}{l}\text { ssc-miR-148b- } \\
5 p\end{array}$ & 671 & 349 & 0.94 & $6.94 \mathrm{E}-07$ & $1.18 \mathrm{E}-05$ \\
\hline $\begin{array}{l}\text { ssc-miR-532- } \\
5 p\end{array}$ & 23,008 & 13,830 & 0.73 & $1.89 \mathrm{E}-06$ & 3.04E-05 \\
\hline $\begin{array}{l}\text { ssc-miR-30a- } \\
5 p\end{array}$ & 16,610 & 10,506 & 0.66 & 3.00E-05 & 0.0003413 \\
\hline ssc-miR-1343 & 641 & 384 & 0.74 & $4.66 \mathrm{E}-05$ & 0.00048942 \\
\hline $\begin{array}{l}\text { ssc-miR-99a- } \\
5 p\end{array}$ & 51,110 & 30,673 & 0.74 & 7.13E-05 & 0.00072047 \\
\hline ssc-miR-30d & 58,223 & 36,038 & 0.69 & 0.00011594 & 0.0010914 \\
\hline $\begin{array}{l}\text { ssc-miR-126- } \\
5 p\end{array}$ & 123 & 61 & 1.02 & 0.00012548 & 0.0011177 \\
\hline
\end{tabular}

Table 5. The top 20 most up-regulated miRNAs at the D8 compared to the D0. 


\begin{tabular}{llllll} 
miRNA & D8 (readcount) & D0 (readcount) & log2FoldChange & pval & padj \\
\hline ssc-miR-708-5p & 4,248 & 1,007 & 2.08 & $1.96 \mathrm{E}-48$ & $6.82 \mathrm{E}-46$ \\
\hline ssc-miR-181a & 44,462 & 10,149 & 2.13 & $3.18 \mathrm{E}-37$ & $5.53 \mathrm{E}-35$ \\
\hline ssc-miR-146b & 2,295 & 197 & 3.54 & $3.74 \mathrm{E}-33$ & $4.33 \mathrm{E}-31$ \\
\hline ssc-miR-126-3p & 2,918 & 1,027 & 1.51 & $1.15 \mathrm{E}-25$ & $1.00 \mathrm{E}-23$ \\
\hline ssc-miR-708-3p & 1,610 & 474 & 1.76 & $6.54 \mathrm{E}-23$ & $4.55 \mathrm{E}-21$ \\
\hline ssc-miR-455-5p & 9,233 & 2,969 & 1.64 & $1.06 \mathrm{E}-20$ & $6.12 \mathrm{E}-19$ \\
\hline ssc-miR-34c & 284 & 81 & 1.81 & $1.22 \mathrm{E}-18$ & $6.07 \mathrm{E}-17$ \\
\hline ssc-miR-455-3p & 1,133 & 379 & 1.58 & $3.42 \mathrm{E}-17$ & $1.49 \mathrm{E}-15$ \\
\hline ssc-miR-181b & 8,339 & 2,860 & 1.544 & $3.94 \mathrm{E}-17$ & $1.52 \mathrm{E}-15$ \\
\hline ssc-miR-378 & 149,879 & 6,304 & 1.25 & $6.19 \mathrm{E}-17$ & $2.15 \mathrm{E}-15$ \\
\hline ssc-miR-378b-3p & 1,356 & 417 & 1.70 & $7.25 \mathrm{E}-16$ & $2.29 \mathrm{E}-14$ \\
\hline ssc-miR-6516 & 388 & 106 & 1.87 & $3.99 \mathrm{E}-12$ & $9.91 \mathrm{E}-11$ \\
\hline ssc-miR-34a & 4,971 & 2,471 & 1.01 & $1.99 \mathrm{E}-10$ & $3.64 \mathrm{E}-09$ \\
\hline ssc-miR-148b-5p & 774 & 358 & 1.11 & $4.87 \mathrm{E}-10$ & $8.08 \mathrm{E}-09$ \\
\hline ssc-miR-199b-5p & 471,495 & 21,615 & 1.13 & $5.94 \mathrm{E}-10$ & $9.40 \mathrm{E}-09$ \\
\hline ssc-miR-148a-3p & 443,734 & 209,486 & 1.08 & $4.70 \mathrm{E}-08$ & $5.64 \mathrm{E}-07$ \\
\hline ssc-miR-423-5p & 7,051 & 4,190 & 0.75 & $1.21 \mathrm{E}-06$ & $1.24 \mathrm{E}-05$ \\
\hline ssc-miR-199a-5p & 354,121 & 201,019 & 0.82 & $2.29 \mathrm{E}-06$ & $2.27 \mathrm{E}-05$ \\
\hline ssc-miR-532-5p & 23,303 & 14,181 & 0.72 & $2.54 \mathrm{E}-06$ & $2.33 \mathrm{E}-05$ \\
\hline ssc-miR-664-5p & 154 & 57 & 1.43 & $2.47 \mathrm{E}-06$ & $2.33 \mathrm{E}-05$ \\
\hline
\end{tabular}

\section{MiRNAs target gene prediction and function pathway analysis}

The nucleotide at 2-8 position of a mature miRNA is called seed region which is critical for binding to the 3 '-UTR of its target genes. To explore the potential regulatory network during intramuscular preadipocyte differentiation, the target genes of differentially expressed known and novel miRNAs were predicted. The target genes predicted of miRNA were shown in Table S8. A total of 130041 corresponding relationships between target genes and miRNA were found in our research.

Based on differentially expressed miRNAs with target genes, significantly GO terms were mainly involved in: (1) metabolic process, single-organism process, intracellular part, intracellular, cell part, cell, cellular component, protein binding, binding, catalytic activity between D4 and D0 (Figure S 7a; Table S9); (2) metabolic process, cellular metabolic process, organic substance metabolic process, intracellular part, 
intracellular, protein binding between D8 and D0 (Figure S 7b; Table S10); (3) cytoplasm, cytoplasmic part, protein binding between D8 and D4 (Figure S 7c; Table S11); Based on differentially expressed miRNAs with target genes, significantly KEGG enrichment were mainly involved in: (1) sphingolipid signaling pathway, regulation of actin cytoskeleton, ras signaling pathway, rap1 signaling pathway, proteoglycans in cancer, PI3K-Akt signaling pathway, pathway in cancer, oxytocin signaling pathway, metabolic pathway, MAPK signaling pathway, HTLV-I infection, focal adhesion, Epstein-Barr virus infetion, endocytosis, chemokine signaling pathway, cAMP signaling pathway, Axon guidance, Apoptosis, AMPK signaling pathway, Adrenergic signaling in cardiomyyocytes between D4 and D0 (Figure 4A); (2) TNF signaling pathway, sphingolipid signaling pathway, regulation of actin cytoskeleton, ras signaling pathway, rap1 signaling pathway, proteoglycans in cancer, PI3K-Akt signaling pathway, Pathways in cancer, NF-kappa B signaling, Metabolic pathways, MAPK signaling pathway, HTLV-1 infection, focal adhesion, endocytosis, cytokine-cytokine receptor interaction, chemokine signaling pathway, axon guidance, apoptosis, AMPK signaling pathway, adrenergic signaling in cardiomyocytes between D8 and D0 (Figure 4B); (3) viral myocarditis, type I diabetes mellitus, transcriptional misregulation in cancer, toxoplasmosis, $T$ cell receptor signaling pathway, staphylococcus aureus infection, rheumatoid arthritis, proteoglycans in cancer, PI3K-Akt signaling pathway, NF-kappa B signaling pathway, malaria, leishmaniasis, intestinal immune network for IgA production, inflammatory bowel disease (IBD), hematopoietic cell lineage, graft-versus-host disease, autoimmune thyroid disease, asthma, antigen processing and presentation, allograft rejection between D8 and D4 (Figure 4C).

\section{Function analysis of common DE genes during intramuscular preadipocyte differentiation}

Based on differentially expressed miRNAs with their target genes, significantly enrichment KEGG terms were commonly involved in: (1) sphingolipid signaling pathway, regulation of actin cytoskeleton, ras signaling pathway rap1 signaling pathway, proteoglycans in cancer, PI3K-Akt signaling pathway, pathway

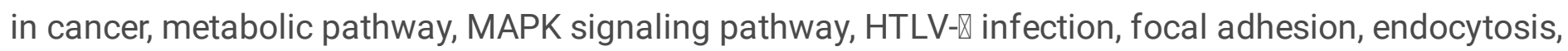
chemokine signaling pathway, axon guidance, apoptosis, AMPK signaling pathway, adrenergic signaling in cardiomyocytes in group D4 vs. D0 and D8 vs. D0 (Figure 4); (2) proteoglycans in cancer, PI3K-Akt signaling pathway in group D4 vs. D0 and D8 vs. D4 (Figure 4); (3) proteoglycans in cancer, PI3K-Akt signaling pathway, NF-kappa B signaling pathway in group D8 vs D0 and D8 vs D4 (Figure 4). Significantly enrichment KEGG terms were commonly involved in proteoglycans in cancer and PI3K-Akt signaling pathway for DE miRNA with their target genes in group D4 vs. D0, D8 vs. D0, and D8 vs. D4 (Figure 4).

\section{Network of miRNAs and mRNAs}

MiRNA is a novel family of non-coding RNAs, and the potential for functionality of most miRNAs is currently uncharacterized. To further investigate whether miRNAs regulate the transcription of their target genes, we performed the network of the miRNA and their target mRNA. A porcine intramuscular preadipocyte development associated ceRNA network containing 85 miRNA-mRNA, 93 miRNA-mRNA, 2 
miRNA-mRNA pairs in group D4 vs D0, D8 vs D0, and D8 vs D4, respectively (Table S12). The ceRNA network may provide a novel perspective for porcine intramuscular preadipocytes (Figure 5).

\section{DE mRNAs of the ceRNA network function analysis}

To uncover the biological mechanisms associated with the development of the porcine intramuscular preadipocyte development, a KEGG pathway enrichment analysis of the DE mRNAs in the ceRNA network was performed. The significant KEGG pathway enrichment included arrhythmogenic right ventricular cardiomyopathy (ARVC), PPAR signaling pathway, phagosome, malaria, lysosome, hematopoietic cell lineage, glycosaminoglycan degradation, fat digestion and absorption, ECM-receptor interaction, AMPK signaling pathway, and adipocytokine signaling pathway (Figure S8).

\section{Validation of RNA-Seq by qRT-PCR}

QRT-PCR was conducted to validate the RNA-Seq data. We randomly selected nine miRNAs (miR-34c, miR-100, miR-126-3p, miR-148a-3p, miR-199b-5p, miR-222, miR-381-3p, miR-455-5p, novel_25) to validate. The RNA samples used in the sequencing were the same as those used in the qRT-PCR. Relative expression levels of genes were calculated based on the mean value from 9 cell samples by using the comparative Ct method. After comparison with the RNA-Seq data, similar expression trends for qRT-PCR were discovered (Figure 6). The qRT-PCR data were shown as the histogram and the $y$-axis on the left, while the RNA-seq data were shown as the line and $y$-axis on the right. Error bars for qRT-PCR data and RNA-seq data were standard errors.

\section{Expression pattern analysis of miR-148a-3p}

In the following, we focused on the differentially expressed and highest expression abundance in D0, D4, and D8 miRNA (ssc-miR-148a-3p). To explore the expression characteristics of miR-148a-3p in different tissues of China Guizhou Congjiang pigs and different differentiation stages of intramuscular preadipocyte. We detected the relative expression level by qRT-PCR (Figure 7). We found that miR-148a-3p was the highest level of expression in the pancreas, followed in the small intestine. The order of tissue relative expression level from high to low was pancreas, small intestine, large intestine, liver, muscle, adipose, spleen, stomach, kidney, heart, and lung (Figure 7A). As shown in figure 7B, the expression level of miR-148a-3p gradually increased from day 0 to day 8 . The expression level of miR-148a-3p on day 2,4 , 6 , and 8 were significantly higher than on day 0 .

\section{Effects of miR-148a-3p on porcine intramuscular preadipocyte proliferation}

To elucidate the potential effect of miR-148a-3p in porcine intramuscular proliferation, cell counting kit-8 (CCK-8), cell cycle assay, 5-Ethynyl-2'-deoxyuridine (EdU), qPCR, and western blotting assays were used. As shown in Figure 8A, synthetic miR-148a-3p mimics, mimics NC, inhibitor, and inhibitor NC were transfected into porcine intramuscular preadipocytes, respectively. As expected, miR-148a-3p mimics significantly increased the expression level of miR-148a-3p compared to mimics NC in porcine intramuscular preadipocytes, while miR-148a-3p inhibitor remarkably decreased the expression level of 
miR-148a-3p compared to inhibitor NC. The CCK-8 assay reveals that transfection miR-148a-3p mimics could significantly increase the total number of preadipocytes compared to the mimics NC group. However, the total number of preadipocytes was significantly decreased by transfection with miR-148a-3p inhibitor compared to inhibitor NC group (Figure 8B). A cell phase assay revealed that miR-148a-3p mimics decreased the proportion of cells in G0/G1 phase and increased the number of preadipocytes in $S$ and G2 phases, while the proportion of cells in the G0/G1 phase significantly increased and decreased the number of preadipocytes in S and G2 phases by transfection miR-148a-3p inhibitor compared to NC group (Figure $8 C$ ). The cell phase result suggests that miR-148a-3p may improve porcine intramuscular preadipocytes proliferation. EdU assay was also indicated that miR-148a-3p overexpression promotes cell proliferation (Figure 8D). Then we also detected the effect of miR-148a-3p on the mRNA expression of cell proliferation-related genes CyclinB, CyclinG 1, CyclinD1, and cyclin-dependent-kinase 2, 3, 4 (CDK2, $C D K 3, C D K 4)$. And found that miR-148a-3p significantly increased the expression of these genes at mRNA levels (Figure 9A, B). The protein expression of proliferating cell nuclear antigen (PCNA), CyclinD1, and $C D K 4$ was analyzed, and also found that miR-148a-3p significantly increased the expression of these genes at protein levels (Figure 9C). These results reveal that miR-148a-3p promotes porcine intramuscular preadipocyte proliferation.

\section{Effects of miR-148a-3p on porcine intramuscular preadipocyte apoptosis}

Hoechst 33342 and propidium iodide (PI), Annexin V-fluorescein isothiocyanate (FITC)/ PI dual staining, and qPCR assays were used to elucidate the potential effect of miR-148a-3p in porcine intramuscular apoptosis. The Annexin V-FITC/PI staining assay showed that miR-148a-3p overexpression inhibited apoptosis in porcine intramuscular preadipocyte (Figure 10A, B). In similar to the finding, miR-148a-3p overexpression significantly decreased the expression levels of Bax and Caspase-3 (Figure 10C). Moreover, we found miR-148a-3p to promote the expression of Bcl-2 which has been described as an antiapoptotic protein (Figure 10C). Hoechst 33342 and propidium iodide (PI) dual staining assays showed that miR-148a-3p significantly decreased the numbers of the dead cells (Figure 10D). These results demonstrate that miR-148a-3p inhibits porcine intramuscular preadipocyte apoptosis.

\section{Effects of miR-148a-3p on porcine intramuscular preadipocyte differentiation}

To assess the effect of miR-148a-3p on porcine intramuscular preadipocyte differentiation, the expression of adipose differentiation markers, hormone-sensitive lipase (HSL), apolipoprotein $E(A P O E)$, lipoprotein lipase ( $L P L)$, fatty acid-binding protein 4 (FABP4), CCAAT/enhancer-binding protein a (CEBPa), peroxisome proliferator-activated receptor $\mathrm{Y}(P P A R Y)$, and fatty acid synthase (FASN) were detected in porcine intramuscular preadipocyte transfection with miR-148a-3p mimics, mimics NC, inhibitor, or inhibitor NC and differentiated for 8 days. We found that mRNA expression of HSL, APOE, LPL, FABP4, $C E B P a, P P A R Y$, and $F A S N$ were significant increased by transfection with miR-148a-3p mimics (Figure $11 \mathrm{~A}$ ), while miR-148a-3p inhibitor remarkably decreased the mRNA expression level of $H S L, A P O E, L P L$, FABP4, CEBPa, PPARY, and FASN (Figure 11B). Overexpression of miR-148a-3p significantly increased the Oil red $O$ staining signal (Figure $11 \mathrm{C}$ ) at day 8 of preadipocyte differentiation. The protein level of $L P L$, 
CEBPa, PPARY were significantly increased in the miR-148a-3p mimic group (Figure 11D). These results demonstrate that miR-148a-3p promotes porcine intramuscular preadipocyte differentiation.

\section{MiR-148a-3p promote porcine intramuscular preadipocyte differentiation by directly targeting PPARGC1A}

The main mechanisms of miRNA participate in many biological processes by negatively regulating target genes through interaction with the $3^{\prime}$-untranslated region (3'-UTR) of the target genes. Therefore, to explore the regulatory mechanism of miR-148a-3p which promotes porcine intramuscular preadipocyte differentiation, we speculated and screened PPARGC1A as a potential target gene of miR-148a-3p by bioinformatics software TargetScan 7.2. We observed that the 3'-UTR region of PPARGC1A mRNA had a binding site for the miR-148a-3p seed sequence (Figure 12A). To confirm this prediction, dual-luciferase reporter assays were performed. We observed that transfection of miR-148a-3p mimics in 293T cells directly repressed the luciferase activities of the wild-type PPARGC1A 3'-UTR reporter when compared to the control group while mutation of the miR-148a-3p binding site in porcine PPARGC1A3'-UTR completely stopped this response (Figure 12B). The protein expression level of PPARGC1A significantly decreased when transfected with miR-148a-3p mimics in porcine intramuscular preadipocytes (Figure 12C). These results suggest that PPARGC1A is a direct target gene of miR-148a-3p during differentiation of porcine intramuscular preadipocytes.

\section{MiR-148a-3p promote porcine intramuscular preadipocyte differentiation by inhibiting AMPK/ACC/CPT-1 signaling pathway}

To further explore the underlying mechanism of how miR-148a-3p promotes porcine intramuscular preadipocyte differentiation, the signal transducer, and the activator of transcription of the AMPK/ACC/CPT-1 signaling pathway was detected at day 8 of adipogenic differentiation. As shown in Figure $13 \mathrm{~A}, \mathrm{~B}, \mathrm{C}$, the levels of $\mathrm{p}-\mathrm{AMPK} / \mathrm{AMPK}, \mathrm{p}-\mathrm{ACC} / \mathrm{ACC}$, and carnitine palmitoyltransferase $1 \mathrm{C}$ (CPT1C) were significantly decreased when over-expressed miR-148a-3p. In contrast, the knockdown miR-148a-3p expression could significantly increase the level of p-AMPK/AMPK, p-ACC/ACC, and CPT1C (Figure 13D, $E, F)$. These results demonstrate that miR-148a-3p promotes porcine intramuscular preadipocyte differentiation by inhibiting the AMPK/ACC/CPT-1 signaling pathway.

Figure 13. MiR-148a-3p promotes porcine intramuscular preadipocyte differentiation through inhibiting the AMPK/ACC/CPT-1 signaling pathway. (A, B) Protein levels of phosphorylated and total AMPK and $A C C$ treated with miR-148a-3p mimic. (C) The protein level of CPT1C treated with miR-148a-3p mimic. (D, E) Protein levels of phosphorylated and total AMPK and ACC treated with miR-148a-3p inhibitor. (F) The protein level of CPT1C was treated with miR-148a-3p inhibitor. All cells were measure at day 8 of adipogenic differentiation. Results were presented as means $\pm S E M, n=3 ;{ }^{\star} p<0.05 ;{ }^{* \star} p<0.01$.

\section{Discussion}

In livestock production, the improvement of the meat quality is crucial, especially the intramuscular fat content [24]\{Du, 2021 \#364\}. Most researches examining the molecular mechanisms in porcine 
intramuscular preadipocyte development focus on protein-coding genes; therefore, studies using highthroughput RNA-seq analysis usually detected in mRNAs [25]. However, the potential function of miRNAs in porcine intramuscular preadipocyte development remains largely unknown. Three critical stages consist of the whole intramuscular preadipocytes differentiation process including growth arrest (D0), mitotic clonal expansion (D4), and late events and terminal differentiation (D8). Therefore, Our present research provides an overview of the types and relative abundances of miRNAs that can be found in porcine intramuscular preadipocyte different differentiation stages (D0, D4, and D8). A total of 16050754, $20667858,18521184,15356301,14585607,16028024,14393598,15354839$, and 13928901 clean reads from d0_1, d0_2, d0_3, d4_1, d4_2, d4_3, d8_1, d8_2 and d8_3 respectively, were obtained through RNAseq, which was sufficient for quantitative analysis of miRNA expression. In present study, we identified 471 miRNAs (TPM $\geq 1)$ as reliably expression genes during intramuscular preadipocyte development. Nevertheless, more than 178 miRNAs were differentially expressed in porcine intramuscular preadipocyte different differentiation stages, which revealed that they may have a developmental stage-specific role in porcine intramuscular preadipocyte. Otherwise, we detected 7 miRNAs that were shared as common differentially expressed genes during the entire differentiation process. The first nucleotide bias of known $20 \mathrm{nt}, 22 \mathrm{nt}, 24 \mathrm{nt}$ miRNAs is $\mathrm{U}$ which was similar to others [12]. While, the first nucleotide bias of known $21 \mathrm{nt}$ and $23 \mathrm{nt}$ miRNA and novel miRNA was different from other researches $[7,8]$. The length of predicted novel miRNA was largely aggregated in $22 \mathrm{nt}$, while the length of known miRNA was mainly clustered in $23 \mathrm{nt}$ which was different from other reports [26]. The different characteristics of miRNA in our present research may because of the different animal species-specific and cell-specific [27], suggesting the classes and expression abundant of miRNA may display spatial and temporal characteristics [28]. Thus, our data may provide a new insight to comprehensively understand the transcriptional regulation mechanism in the porcine intramuscular preadipocyte of Guizhou Congjiang pig.

To explore the potential regulatory function affecting intramuscular preadipocytes, the target genes of DE miRNAs were predicted. Thereafter, GO and KEGG pathway analysis was performed with DE miRNAregulated target genes. As expected, many classical functional categories such as cellular component, single-organism process, metabolic process, organic substance metabolic process, cytoplasm, cytoplasmic part, and protein binding were detected. These functional groups might play critical roles in intramuscular preadipocyte differentiation [29]. We found that the PI3K-Akt signaling pathway was commonly enriched in group D4 vs. D0, D8 vs. D0, and D8 vs. D4. PI3K-Akt signaling pathway plays a pivotal role in lipid metabolism, glucose metabolism, and cell development, regulated by insulin receptor substrate [30]. The signaling pathway, including regulation of actin cytoskeleton, metabolic pathway, MAPK signaling pathway, and AMPK signaling pathway, were significantly enriched during intramuscular preadipocyte differentiation. This result was similar to previous researched $[28,31,32]$ and may be related to the development of intramuscular preadipocyte [33]. Unexpectedly, the related-disease signaling pathway, including proteoglycans in cancer, pathway in cancer, and HTLV- $₫$ infection, were enriched which may give us a new view to comprehensively understand the mechanism of miRNA in diseases [34, 35].

Based on our observation of several miRNAs being differentially expressed across intramuscular preadipocyte differentiation, we propose that studying miRNAs may lead to the discovery of a large 
number of regulatory miRNAs involved in porcine intramuscular preadipocyte proliferation and differentiation. In our present study, we exemplify the involvement of one miRNA (miR-148a-3p) in regulating intramuscular preadipocyte proliferation, differentiation, and apoptosis. We found that miR148a-3p expression level was increased during intramuscular preadipocyte differentiation, indicating that miR-148a-3p might regulate intramuscular preadipocyte adipogenesis.

Cell-cycle entry in the early $\mathrm{G} 1$ phase relies on the activation of $C D K 4$ and $C D K 6$ through combing with cyclin $\mathrm{D}[36,37]$. Cyclin-dependent kinases 2 (CDK2) have been recognized as key regulators of cell growth and proliferation and essential for $\mathrm{G} 1$ to $\mathrm{S}$ phase transition by binding of cyclin $\mathrm{E}[38,39]$. Cyclin $G$ can associate with $C D K 5$ and serves as a negative regulator of $p 53$ by activating $M d m 2$ through dephosphorylation to improve cell proliferation [40]. The gain and loss-of-function studies in porcine intramuscular preadipocytes were applied to detect the function of miR-148a-3p in porcine intramuscular preadipocytes. The genes and protein expression level assay showed that overexpression of miR-148a-3p promoted the expression of cell cycle-related genes, including CDK2, CDK3, CDK4, CCNB, CCNG1, and $C C N D 1$, and significantly accelerated the cell cycle progression by decreasing the proportion of cells in G0/G1 phase by flow cytometry. The proliferation of bone marrow mesenchymal stem cell and non-smallcell lung cancer can also be promoted by miR-148a-3p mimic [41, 42] which consistent with our present research. However, Bao et al. and Wang et al. have reported that overexpression of miR-148a-3p inhibited the proliferation of gastric cancer cells [43] and diabetic retinopathy cell model [44], respectively. Therefore, miR-148a-3p displays inconsistent effects in the proliferation of different cell types. The discrepancy of the function of miR-148a-3p in different cell lines may because of miRNA-mRNA targeting and the interplay relationship that differs among tissue and cell types $[6,10]$.

MiRNAs have a profound impact on many processes including cell proliferation, apoptosis, stress responses, maintenance of stem cell potency and metabolism. A lot of miRNAs have been shown to have an impact on cell proliferation, such as miR-34, miR-14-92, miR-372-373, and miR-155 [45]. Caspases and the $\mathrm{BCl}-2$ family are the two most important groups of proteins that participate in all pathways of apoptotic [46]. The flow cytometry, PI dual staining and apoptosis-related genes expression level assay showed that miR-148a-3p promotes apoptosis in porcine intramuscular preadipocyte. However, previous research reported that miR-148a-3p decreased apoptosis in the human retinal microvascular endothelial cell by targeting TGFB2 and FGF2 [44]. The difference function of miR-148a-3p in cell apoptosis might associate with cell type and the target genes $[47,48]$.

In past years, many studies have proved that miRNAs play key roles in regulating adipogenesis $[4,49]$. In adipogenesis, miR-425-5p, miR-540, miR-98, and miR-125a-5p inhibited adipogenic differentiation $[4,29$, 50,51 ], while miR-146b, miR-223, and miR-145 promoted adipogenic differentiation [52-54]. In present study, we explored the physiological role of miR-148a-3p in porcine intramuscular preadipocyte differentiation. We found that miR-148a-3p level was increased during porcine intramuscular preadipocyte differentiation, indicating that miR-148a-3p might regulate intramuscular preadipocyte adipogenesis. We found that the protein expression level of PPARYCEBPA and $\angle P L$ increased in cells transfected with miR-148a-3p mimics. Consistently, inhibiting of miR-148a-3p expression declined 
intramuscular preadipocyte differentiation. These data suggest that miR-148a-3p is a positive regulator for porcine intramuscular preadipocyte adipogenesis.

Previously, several studies demonstrated that miRNA takes part in many biological processes by negatively regulating target genes through interaction with the $3^{\prime}$-untranslated regions ( 3 '-UTR) of the target mRNAs [6]. To further explore the molecular mechanisms that miR-148a-3p promotes porcine intramuscular preadipocytes differentiation, we predicted target genes of miR-148a-3p using TargetScan 7.2. Among the potential target genes, we observed that the $3^{\prime}$-UTR region of proliferator-activated receptor gamma coactivator 1-alpha (PPARGC1A) mRNA had a binding site for miR-148a-3p seed sequences. Luciferase reporter assays and western blot experiment were confirmed that miR-148a-3p is a direct target gene of miR-148a-3p during differentiation of porcine intramuscular preadipocyte. The PPARGC1A is a nuclear hormone receptor coactivator peroxisome that regulates the gluconeogenesis, lipid metabolism, energy metabolism, and detoxification of reactive oxygen species produced by mitochondria $[55,56]$. In recent years, a total of reporters have been proved that PPARGC1A might promote insulin resistance [57], regulate IRS1:IRS2 ratio [55], and lipid oxidation [58]. Specifically, PPARGC1A has been proved to be a key fatty acid oxidation factor through regulating the fatty acid oxidation signaling pathway [59].

In present study, we showed that miR-148a-3p could target PPARGC1A, which is associated with fatty acid oxidation [59]. So, we further researched a mechanism for the promotion function of miR-148a-3p in porcine intramuscular preadipocyte adipogenesis through the fatty acid oxidation pathway. 5'Adenosine monophosphate (AMP)-activated protein kinase (AMPK) is a highly adaptive complex that as a biologic sensor and becomes active in response to the elevated AMP/ATP ratio via attachment to adenosine diphosphate and/or AMP [60]. AMPK can suppress the lipogenic gene expression and triglyceride synthesis and accumulation via the phosphorylation of ACC and CPT1C pathways [61]. Therefore, AMPK/ACC/CPT1C signaling pathway plays an important role in the regulation of fatty acid metabolism [62]. In a diet-induced obese mice model, Hakka stir-fried tea could effectively ameliorate obesity and related metabolic disorder by the activation of the AMPK/ACC/CPT1 pathway in the liver [63]. In an insulin resistance C57BL/6J mice model, Yin Zhi Huang has associated with a decrease of AMPK/SREBP-1 pathway-mediated de novo lipogenesis and an increase of AMPK/ACC/CPTI pathwaymediated mitochondrial fatty acid $\beta$ oxidation to ameliorate insulin resistance and facilitate lipid metabolism [64]. In current study, we found that miR-148a-3p inhibited AMPK to phosphorylate ACC activating its' ability to convert malonyl-CoA from acetyl-CoA consequently decreased CPT1C content to reduce mitochondrial fatty acid oxidation. These data were also parallel to the degree of increased adipose differentiation markers. These data suggest that miR-148a-3p can positive regulator for porcine intramuscular preadipocyte adipogenesis by decreased the AMPK/ACC/CPTIC pathway-mediated mitochondrial fatty acid $\beta$ oxidation.

\section{Conclusions}


In summary, our study provides a catalog of miRNA expression during the porcine intramuscular preadipocyte differentiation. Numbers of miRNAs were annotated, several of which present a highly different abundance in different differentiation stages. We further characterized an abundant miRNA, miR-148a-3p, to promote proliferation and differentiation during intramuscular adipogenesis in Chinese Guizhou Congjiang Pigs Breeds by targeting PPARGC1A. We anticipate that these results will be a stepping stone to identifying the genetic mechanisms governing intramuscular adipogenesis formation and regeneration, which may be implemented improvement of meat quality.

\section{Abbreviations}

PPARGC1A: peroxisome proliferator-activated receptor gamma coactivator 1 alpha; IMF: Intramuscular fat; PBS: Phosphate buffered saline; FBS: Fetal bovine serum; ncRNA: non-coding RNA; miRNA: microRNA; RIN: RNA integrity number; RT-qPCR: Quantitative real-time PCR; UTR: Untranslated region; RNA-seq: High-through put sequencing of RNA; DE genes: differentially expressed genes; CCNB: Cyclin B; CCNG1: Cyclin G1; CCND1: Cyclin D1; CDK2: Cyclin-dependent kinase 2; CDK3: Cyclin-dependent kinase 3; CDK4: Cyclin-dependent kinase 4; PPARy: peroxisome proliferator-activated receptor gamma; FABP4: Fatty acid-binding protein 4; HSL: Hormone-sensitive lipase, HSL; APOE: Apolipoprotein E; LPL: Lipoprteinlipase; CEBPa: CCAAT/enhancer binding protein alpha; AMPK: Adenosine 5'-monophosphateactivated protein kinase; ACC: Acetyl-CoA carboxylase; CPT1C: Carnitine palmitoyltransferase 1C.

\section{Declarations}

\section{Acknowledgments}

We would like to thank the research assistants and laboratory technicians who contributed to this study.

\section{Author' contributions}

XHQ and TLL conceived and designed the experimental plan. TLL, CZJ, TMD, CB collected samples and performed the experiments. TLL and XHQ drafted and revised this manuscript. All authors read and approved the final manuscript.

\section{Funding}

This work was supported by the National Science and Technology Support Program (2015BAD03B02-3); the National Natural Science Foundation of China (Grant No. 32060759); the National Natural Science Foundation of Guizhou Province (Grant No. ZK[2021] general 165); the subsidy project from NSFC of Guizhou Academy of Agricultural Sciences (NO.[2021]63) and the Fund of Guizhou Animal Husbandry and Veterinary Research Institute (No. [2018]03-03).

\section{Availability of data and materials}


The data analyzed during the current study are available from the corresponding author on reasonable request.

\section{Ethics approval and consent to participate}

All experimental procedures were approved by the Guizhou University Animal Care and Use Committee, Guizhou, China.

\section{Consent for publication}

Not applicable

\section{Competing interests}

The authors declare that they have no competing interests.

\section{References}

[1] Hocquette JF, Gondret F, Baeza E, Medale F, Jurie C, Pethick DW. Intramuscular fat content in meatproducing animals: development, genetic and nutritional control, and identification of putative markers. Animal. 2010; 4(2): 303-319.

[2] Dodson MV, Allen RE, Du M, Bergen WG, Velleman SG, Poulos SP, Fernyhough-Culver M, Wheeler MB, Duckett SK, Young MR. Invited review: Evolution of meat animal growth research during the past 50 years: Adipose and muscle stem cells. J Anim Sci. 2015; 93(2): 457-481.

[3] Sun WX, Dodson MV, Jiang ZH, Yu SG, Chu WW, Chen J. Myostatin inhibits porcine intramuscular preadipocyte differentiation in vitro. Domest Anim Endocrinol. 2016; 55(2016): 25-31.

[4] Chen FF, Xiong Y, Peng Y, Gao Y, Qin J, Chu GY, Pang WJ, Yang GS. MiR-425-5p inhibits differentiation and proliferation in porcine intramuscular preadipocytes. Int J Mol Sci. 2017; 18: 1422-1467.

[5] Ambele MA, Dessels C, Durandt C, Pepper MS. Genome-wide analysis of gene expression during adipogenesis in human adipose-derived stromal cells reveals novel patterns of gene expression during adipocyte differentiation. Stem Cell Res. 2016; 16(3): 725-734.

[6] Bartel DP. Metazoan microRNAs. Cell. 2018; 173(1): 20-51.

[7] Liu J, Ning C, Li B, Li R, Wu W, Liu H. Hepatic microRNAome reveals potential microRNA-mRNA pairs association with lipid metabolism in pigs. Asian-Australas J Anim Sci 2019; 32(9): 1458-1468.

[8] Liu X, Wei H, Liao S, Ye J, Zhu L, Xu Z. MicroRNA transcriptome analysis of porcine vital organ responses to immunosuppressive porcine cytomegalovirus infection. Virol J. 2018; 15(1): 16-32. 
[9] Seiri P, Abi A, Soukhtanloo M. PPAR-y: Its ligand and its regulation by microRNAs. J Cell Biochem. 2019; 120(7): 10893-10908.

[10] Gebert LFR, MacRae IJ. Regulation of microRNA function in animals. Nat Rev Mol Cell Biol. 2019; 20(1): 21-37.

[11] Romao JM, Jin W, Dodson MV, Hausman GJ, Moore SS, Guan LL. MicroRNA regulation in mammalian adipogenesis. Exp Biol Med. (Maywood). 2011; 236(9): 997-1004.

[12] Wei W, Li B, Liu K, Jiang A, Dong C, Jia C, Chen J, Liu H, Wu W. Identification of key microRNAs affecting drip loss in porcine longissimus dorsi by RNA-Seq. Gene. 2018; 647: 276-282.

[13] Tan Z, Du J, Shen L, Liu C, Ma J, Bai L, Jiang Y, Tang G, Li M, Li X. miR-199a-3p affects adipocytes differentiation and fatty acid composition through targeting SCD. Biochem Biophys Res Commun. 2017(1); 492: 82-88.

[14] Wang W, Cheng M, Qiao S, Wang Y, Li H, Wang N. Gga-miR-21 inhibits chicken pre-adipocyte proliferation in part by down-regulating kruppel-like factor 5. Poult Sci. 2017; 96(1): 200-210.

[15] Zhang P, Du J, Wang L, Niu L, Zhao Y, Tang G, Jiang Y, Shuai S, Bai L, Li X, Wang J, Zhang S, Zhu L. MicroRNA-143a-3p modulates preadipocyte proliferation and differentiation by targeting MAPK7. Biomed Pharmacother. 2018; 108(2018): 531-539.

[16] Qi R, Wang J, Wang Q, Qiu X, Yang F, Liu Z, Huang J. MicroRNA-425 controls lipogenesis and lipolysis in adipocytes. Biochim Biophys Acta Mol Cell Biol Lipids. 2019; 1864(5): 744-755.

[17] Langmead B, Trapnell C, Pop M, Salzberg SL. Ultrafast and memory-efficient alignment of short DNA sequences to the human genome. Genome Biol. 2009; 10(3): R25.

[18] Friedlander MR, Mackowiak SD, Li N, Chen W, Rajewsky N. miRDeep2 accurately identifies known and hundreds of novel microRNA genes in seven animal clades. Nucleic Acids Res. 2012; 40(1): 37-52.

[19] Wen M, Shen Y, Shi S, Tang T. miREvo: an integrative microRNA evolutionary analysis platform for next-generation sequencing experiments. BMC Bioinformatics. 2012; 13: 140-150.

[20] Anders S, Huber W. Differential expression analysis for sequence count data. Genome Biol. 2010; 11(10): R106.

[21] Young MD, Wakefield MJ, Smyth GK, Oshlack A. Gene ontology analysis for RNA-seq: accounting for selection bias. Genome Biol. 2010; 11(2): R14.

[22] Mao X, Cai T, Olyarchuk JG, Wei L. Automated genome annotation and pathway identification using the KEGG Orthology (KO) as a controlled vocabulary. Bioinformatics. 2005; 21(19): 3787-3793. 
[23] Shannon P, Markiel A, Ozier O, Baliga NS, Wang JT, Ramage D, Amin N, Schwikowski B, Ideker T. Cytoscape: a software environment for integrated models of biomolecular interaction networks. Genome Res. 2003; 13(11): 2498-2504.

[24] Larzul C. How to improve meat quality and welfare in entire male pigs by genetics. Animals (Basel). 2021; 11(3): 699-715.

[25] Li B, Dong C, Li P, Ren Z, Wang H, Yu F, Ning C, Liu K, Wei W, Huang R, Chen J,Wu W, Liu $\mathrm{H}$. Identification of candidate genes associated with porcine meat color traits by genome-wide transcriptome analysis. Sci Rep. 2016; 6(1): 35224-35236.

[26] Bao H, Kommadath A, Liang G, Sun X, Arantes AS, Tuggle CK, Bearson SMD, Plastow GS, Stothard P, Guan LL. Genome-wide whole blood microRNAome and transcriptome analyses reveal miRNA-mRNA regulated host response to foodborne pathogen Salmonella infection in swine. Sci Rep. 2015; 5(1): $12620-12632$.

[27] Goody D, Pfeifer A. MicroRNAs in brown and beige fat. Biochim Biophys Acta Mol Cell Biol Lipids. 2019; 1864(1): 29-36.

[28] Li G, Li Y, Li X, Ning X, Li M., Yang G. MicroRNA identity and abundance in developing swine adipose tissue as determined by solexa sequencing. J Cell Biochem. 2011;112(5): 1318-1328.

[29] Du J, Xu Y, Zhang P, Zhao X, Gan M, Li Q, Ma J, Tang G, Jiang Y, Wang J, Li X, Zhang S, Zhu L. MicroRNA-125a-5p affects adipocytes proliferation, differentiation and fatty acid composition of porcine intramuscular fat. Int J Mol Sci. 2018; 19(2): 501-516.

[30] Zhou YJ, Xu N, Zhang XC, Zhu YY, Liu SW, Chang YN. Chrysin improves glucose and lipid metabolism disorders by regulating the AMPK/PI3K/AKT signaling pathway in insulin-resistant HepG2 cells and HFD/STZ-induced C57BL/6J mice. J Agric Food Chem. 2021; 69(20): 5618-5627.

[31] Hao Y, Liu JR, Zhang Y, Yang PG, Feng YJ, Cui YJ, Yang CH, Gu XH. The microRNA expression profile in porcine skeletal muscle is changed by constant heat stress. Anim Genet. 2016; 47(3): 365-369.

[32] Xi Y, Liu H, Zhao Y, Li J, Li W, Liu G, Lin J, Liu W, Zhang J, Lei M, Ni D. Comparative analyses of longissimus muscle miRNAomes reveal microRNAs associated with differential regulation of muscle fiber development between Tongcheng and Yorkshire pigs. PLoS One. 2018; 13(7): e0200445.

[33] Oclon E, Latacz A, Zubel-Lojek J, Pierzchala-Koziec K. Hyperglycemia-induced changes in miRNA expression patterns in epicardial adipose tissue of piglets. J Endocrinol. 2016; 229(3): 259-266.

[34] Razavi ZS, Asgarpour K, Mahjoubin-Tehran M, Rasouli S, Khan H, Shahrzad MK, Hamblin MR, Mirzaei H. Angiogenesis-related non-coding RNAs and gastrointestinal cancer. Mol Ther Oncolytics. 2021; 21(2372-7705): 220-241. 
[35] Xing Y, Ruan G, Ni H, Qin H, Chen S, Gu X, Shang J, Zhou Y, Tao X, Zheng

$\mathrm{L}$. Tumor immune microenvironment and its related miRNAs in tumor progression. Front Immunol. 2021; 12(1664-3224): 624725-624741.

[36] Pack LR, Daigh LH, Chung M, Meyer T. Clinical CDK4/6 inhibitors induce selective and immediate dissociation of p21 from cyclin D-CDK4 to inhibit CDK2. Nat. Commun. 2021; 12(1): 3356-3368.

[37] Ekholm SV, Reed SI. Regulation of G1 cyclin-dependent kinases in the mammalian cell cycle. Curr Opin Cell Biol. 2000; 12(6): 676-684.

[38] Ohtsubo M, Theodoras AM, Schumacher J, Roberts JM, Pagano M. Human cyclin E, a nuclear protein essential for the G1-to-S phase transition. Mol Cell Biol. 1995; 15(5): 2612-2624.

[39] Vladar EK, Stratton MB, Saal ML, Salazar D, Simone G, Wang X, Wolgemuth D, Stearns T, Axelrod JD. Cyclin-dependent kinase control of motile ciliogenesis. Elife. 2018; 7(2050-084X): e36375.

[40] Chen X. Cyclin G: a regulator of the p53-Mdm2 network. Dev Cell. 2002; 2(5): 518-519.

[41] Huang S, Li Y, Wu P, Xiao Y, Duan N, Quan J, Du W. microRNA-148a-3p in extracellular vesicles derived from bone marrow mesenchymal stem cells suppresses SMURF1 to prevent osteonecrosis of femoral head. J Cell Mol Med. 2020; 24(19): 11512-11523.

[42] Su H, Fan G, Huang J, Qiu X. YBX1 regulated by runx3-miR-148a-3p axis facilitates non-small-cell lung cancer progression. Cell Signal. 2021; 85(1873-3913): 110049-110051.

[43] Bao C, Guo L. MicroRNA-148a-3p inhibits cancer progression and is a novel screening biomarker for gastric cancer. J Clin Lab Anal. 2020; 34(10): e23454.

[44] Wang J, Yao Y, Wang K, Li J, Chu T, Shen H. MicroRNA-148a-3p alleviates high glucose-induced diabetic retinopathy by targeting TGFB2 and FGF2. Acta Diabetol. 2020; 57(12): 1435-1443.

[45] He L, He X, Lowe SW, Hannon GJ. microRNAs join the p53 network-another piece in the tumoursuppression puzzle. Nat Rev Cancer. 2007; 7: 819-822.

[46] Grilo AL, Mantalaris A. Apoptosis: A mammalian cell bioprocessing perspective. Biotechnol Adv. 2019; 37(3): 459-475.

[47] Pajvani UB, Trujillo ME, Combs TP, lyengar P, Jelicks L, Roth KA, Kitsis RN, Scherer PE. Fat apoptosis through targeted activation of caspase 8: a new mouse model of inducible and reversible lipoatrophy. Nat Med. 2005; 11(7): 797-803.

[48] Wang SS, Wang C, Chen $\mathrm{H}$. MicroRNAs are critical in regulating smooth muscle cell mineralization and apoptosis during vascular calcification. J Cell Mol Med. 2020; 24(23): 13564-13572. 
[49] Kajimoto K, Naraba H, Iwai N. MicroRNA and 3T3-L1 pre-adipocyte differentiation. RNA. 2006; 12(9): 1626-1632.

[50] Chen L, Chen Y, Zhang S, Ye L, Cui J, Sun Q, Li K, Wu H, Liu L. MiR-540 as a novel adipogenic inhibitor impairs adipogenesis via suppression of PPARY. J Cell Biochem. 2015; 116(6): 969-976.

[51] Dai Y, Wu X, Dai D, Li J, Mehta JL. MicroRNA-98 regulates foam cell formation and lipid accumulation through repression of LOX-1. Redox Biol. 2018; 16(2213-2317): 255-262.

[52] Chen L, Dai YM, Ji CB, Yang L, Shi CM, Xu GF, Pang LX, Huang FY, Zhang CM, Guo XR. MiR-146b is a regulator of human visceral preadipocyte proliferation and differentiation and its expression is altered in human obesity. Mol Cell Endocrinol. 2014; 393(1-2): 65-74.

[53] Guan X, Gao Y, Zhou J, Wang J, Zheng F, Guo F, Chang A, Li X, Wang B. miR-223 regulates adipogenic and osteogenic differentiation of mesenchymal stem cells through a C/EBPs/miR-

223/FGFR2 regulatory feedback loop. Stem Cells. 2015; 33(5): 1589-1600.

[54] Guo Y, Chen Y, Zhang Y, Zhang Y, Chen L, Mo D. Up-regulated miR-145 expression inhibits porcine preadipocytes differentiation by targeting IRS1. Int J Biol Sci. 2012; 8(10): 1408-1417.

[55] Besse-Patin A, Jeromson S, Levesque-Damphousse P, Secco B, Laplante M, Estall JL. PGC1A regulates the IRS1:IRS2 ratio during fasting to influence hepatic metabolism downstream of insulin. Proc Natl Acad Sci USA. 2019; 116(10): 4285-4290.

[56] Puigserver P, Wu Z, Park CW, Graves R, Wright M, Spiegelman BM. A cold-inducible coactivator of nuclear receptors linked to adaptive thermogenesis. Cell. 1998; 92(6): 829-839.

[57] Koo SH, Satoh H, Herzig S, Lee CH, Hedrick S, Kulkarni R, Evans RM. Olefsky J, Montminy M. PGC1 promotes insulin resistance in liver through PPAR-alpha-dependent induction of TRB-3. Nat Med. 2004; 10(5): 530-534.

[58] Puigserver P. Tissue-specific regulation of metabolic pathways through the transcriptional coactivator PGC1-alpha. Int J Obes (Lond). 2005; 29(0307-0565): S5-9.

[59] Hou N, Huang Y, Cai SA, Yuan WC, Li LR, Liu XW, Zhao GJ, Qiu XX, Li AQ, Cheng CF, Liu SM, Chen XH, Cai DF, Xie JX, Chen MS, Luo CF. Puerarin ameliorated pressure overload-induced cardiac hypertrophy in ovariectomized rats through activation of the PPARalpha/PGC-1 pathway. Acta Pharmacol Sin. 2021; 42(1): 55-67.

[60] Dehnavi S, Kiani A, Sadeghi M, Biregani AF, Banach M, Atkin SL, Jamialahmadi T, Sahebkar A. Targeting AMPK by statins: a potential therapeutic approach. Drugs. 2021; 81(8): 923-933. 
[61] Pang Y, Xu X, Xiang X, Li Y, Zhao Z, Li J, Gao S, Liu Q, Mai K, Ai Q. High fat activates OGIcNAcylation and affects AMPK/ACC pathway to regulate lipid metabolism. Nutrients. $2021 ; 13(6)$ : 1740-1754.

[62] Ho C, Gao Y, Zheng D, Liu Y, Shan S, Fang B, Zhao Y, Song D, Zhang Y, Li Q. Alisol A attenuates high-fat-diet-induced obesity and metabolic disorders via the AMPK/ACC/SREBP-1c pathway. J Cell Mol Med. 2019; 23(8): 5108-5118.

[63] Li Q, Lai X, Sun L, Cao J, Ling C, Zhang W, Xiang L, Chen R, Li D, Sun S. Antiobesity and antiinflammation effects of Hakka stir-fried tea of different storage years on high-fat diet-induced obese mice model via activating the AMPK/ACC/CPT1 pathway. Food Nutr Res. 2020; 64(1654-661X): 1681-1694.

[64] Yao Q, Li S, Cheng X, Zou Y, Shen Y, Zhang S. Yin Zhi Huang, a traditional Chinese herbal formula, ameliorates diet-induced obesity and hepatic steatosis by activating the AMPK/SREBP-1 and the AMPK/ACC/CPT1A pathways. Ann Transl Med. 2020; 8(2305-5839): 231-244.

\section{Figures}


A.

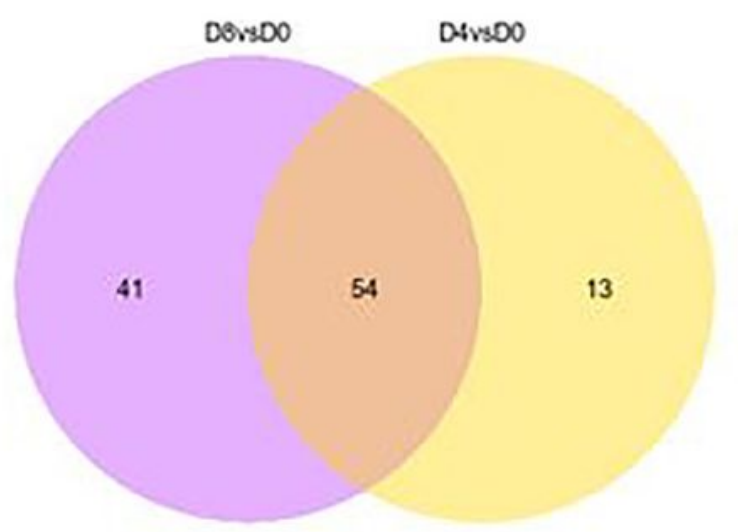

C.

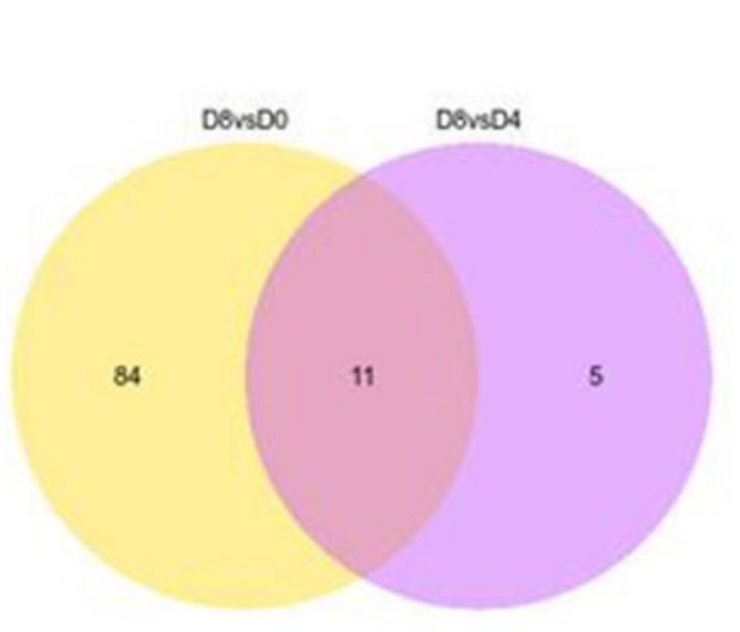

B.

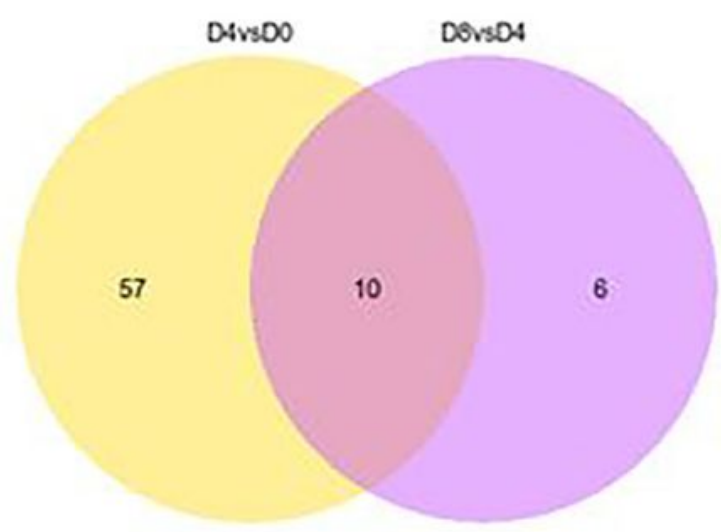

D.
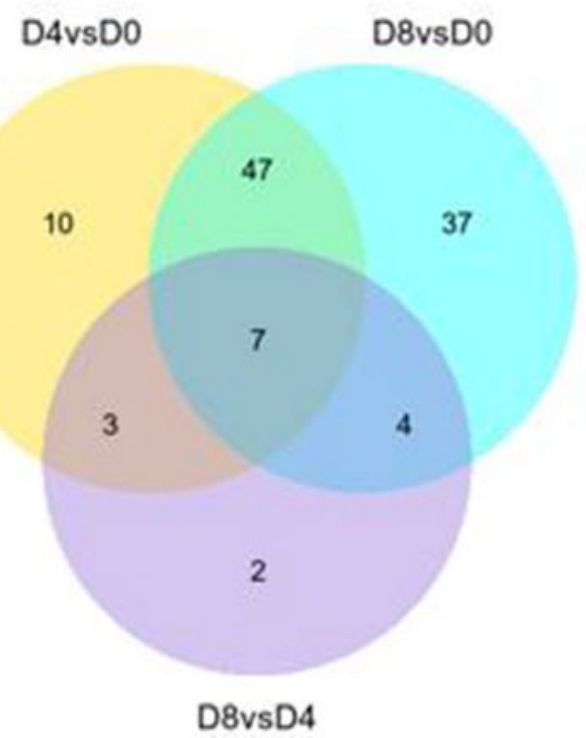

\section{Figure 1}

Venn diagram of DE miRNAs at different time-point comparisons. 
A.

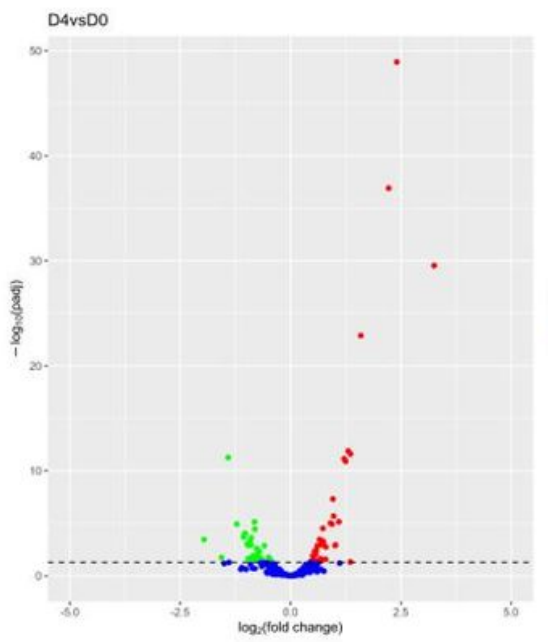

B.

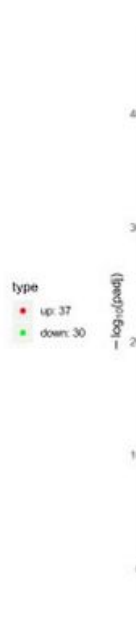

C.

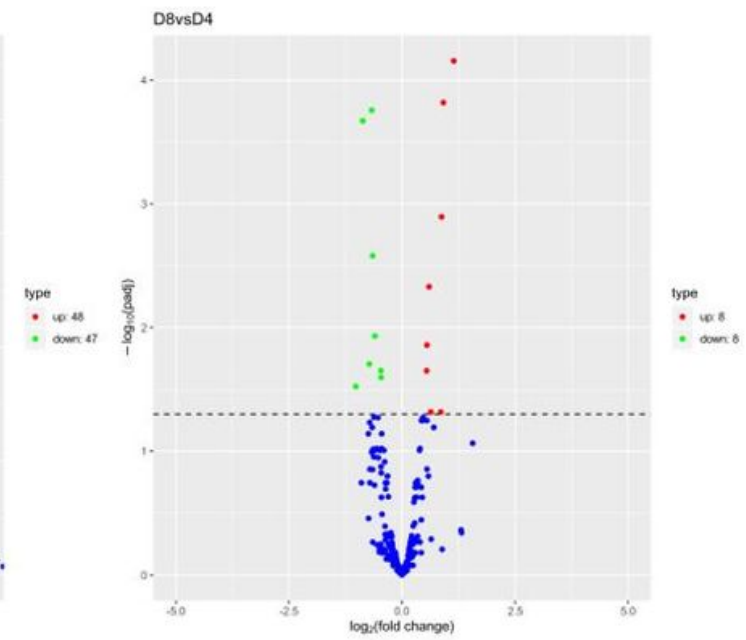

Figure 2

Volcano plot of differentially expressed miRNAs in D4 vs. D0 (A), D8 vs D0 (B), and D8 vs D4 (C). Blue points represent the insignificant genes, red points represent the upregulated genes, and green points represent the downregulated genes, and padj凶0.05. 


\section{Cluster analysis of differentially expressed sRNA}

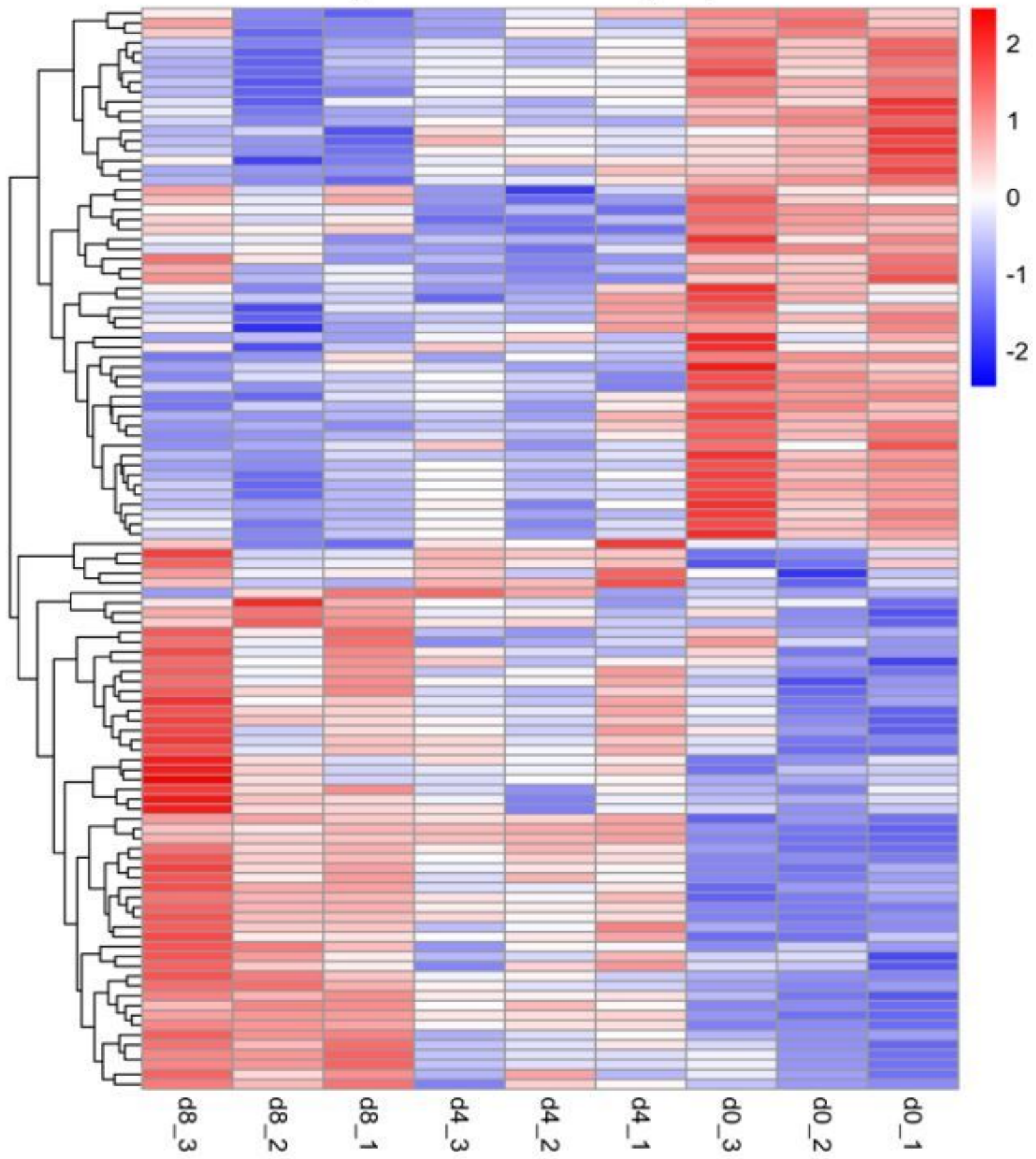

Figure 3

The heatmap of DE miRNA during intramuscular preadipocyte differentiation. 
A.

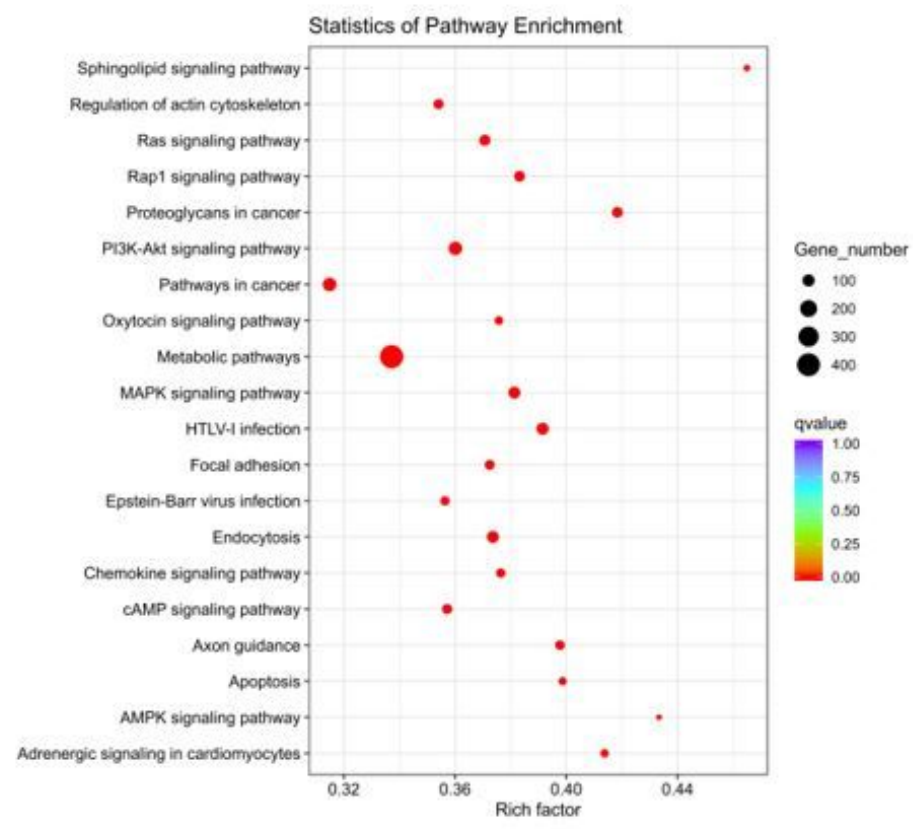

C.

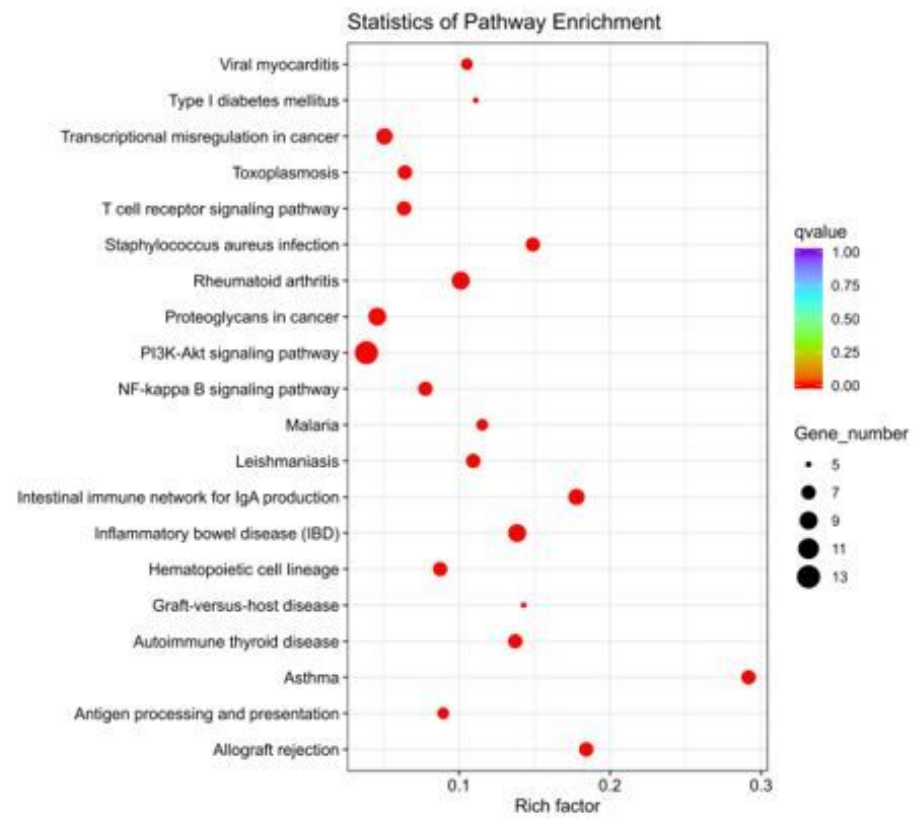

B.

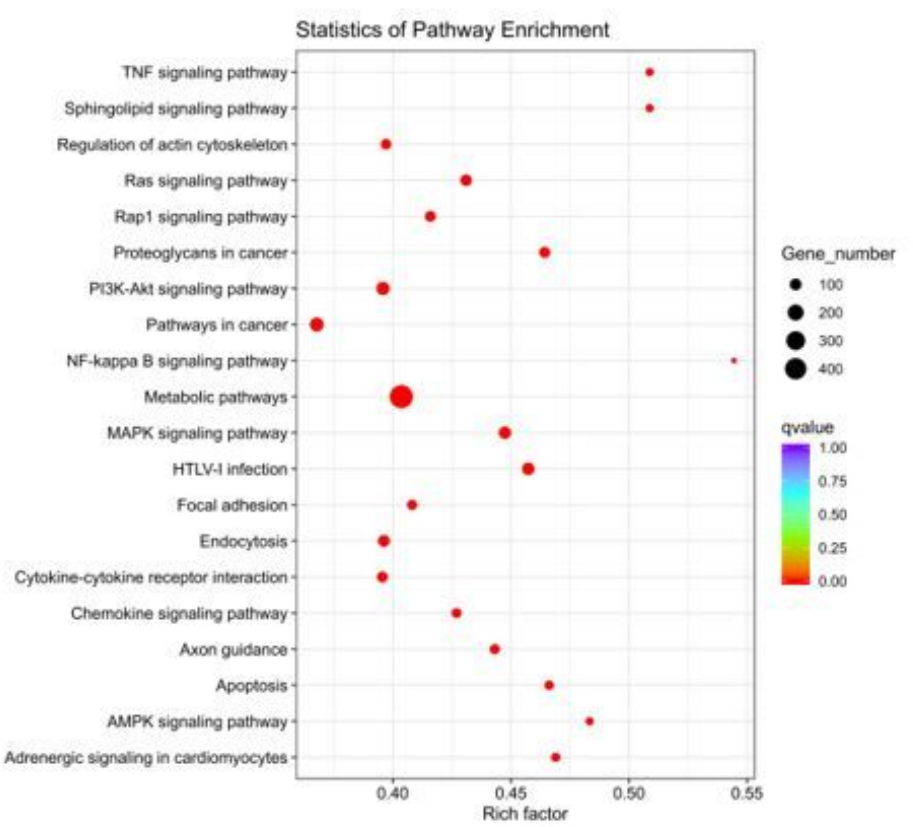

\section{Figure 4}

Kyoto Encyclopedia of Genes and Genomes (KEGG) enrichment analysis of differentially expressed miRNAs with their target genes in group D4 vs D0 (A), D8 vs D0 (B), and D8 vs D4 (C). 
B.
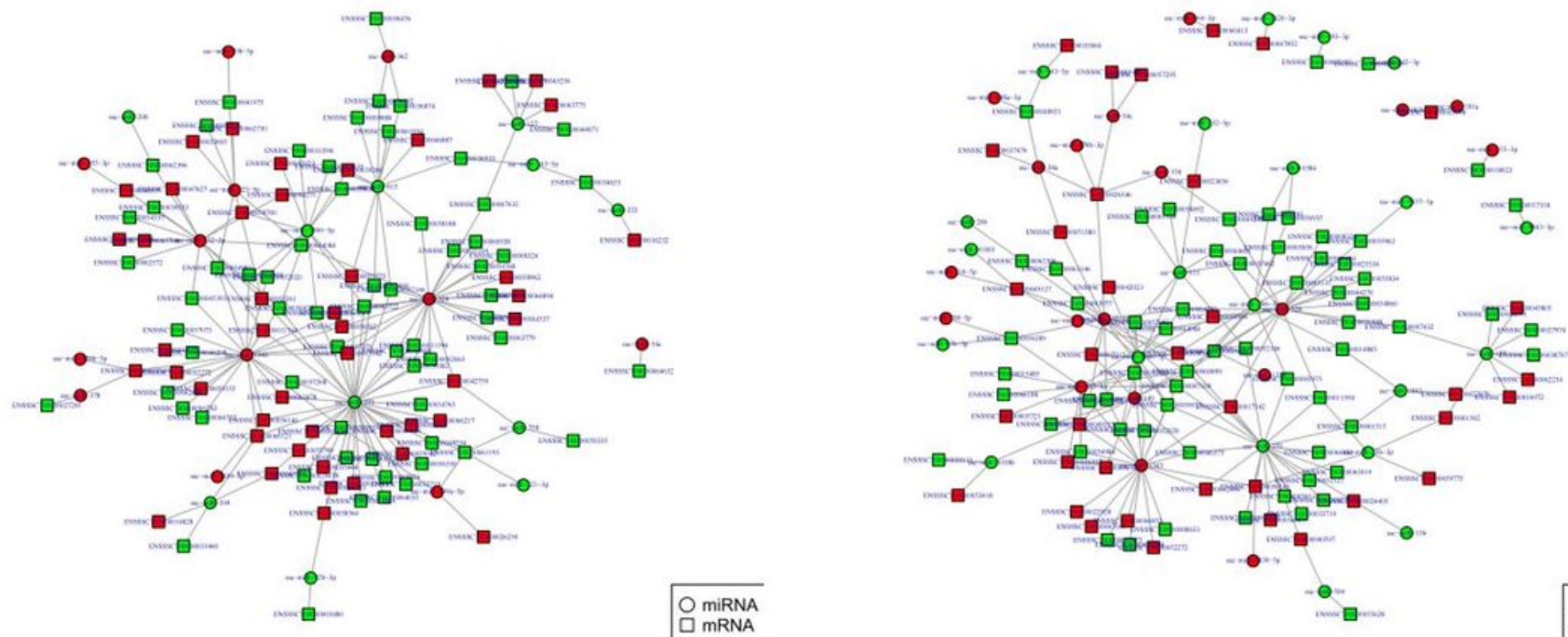

O MiRNA

mRNA
mRNA mRNA

\section{Figure 5}

Co-expression network in porcine intramuscular preadipocyte of the miRNA-mRNA in group D4 vs D0 (A) and D8 vs D0 (B). The red nodes represent the upregulated genes, and the blue nodes represent the downregulated genes. The circle and quadrate represent miRNAs and mRNA, respectively. 

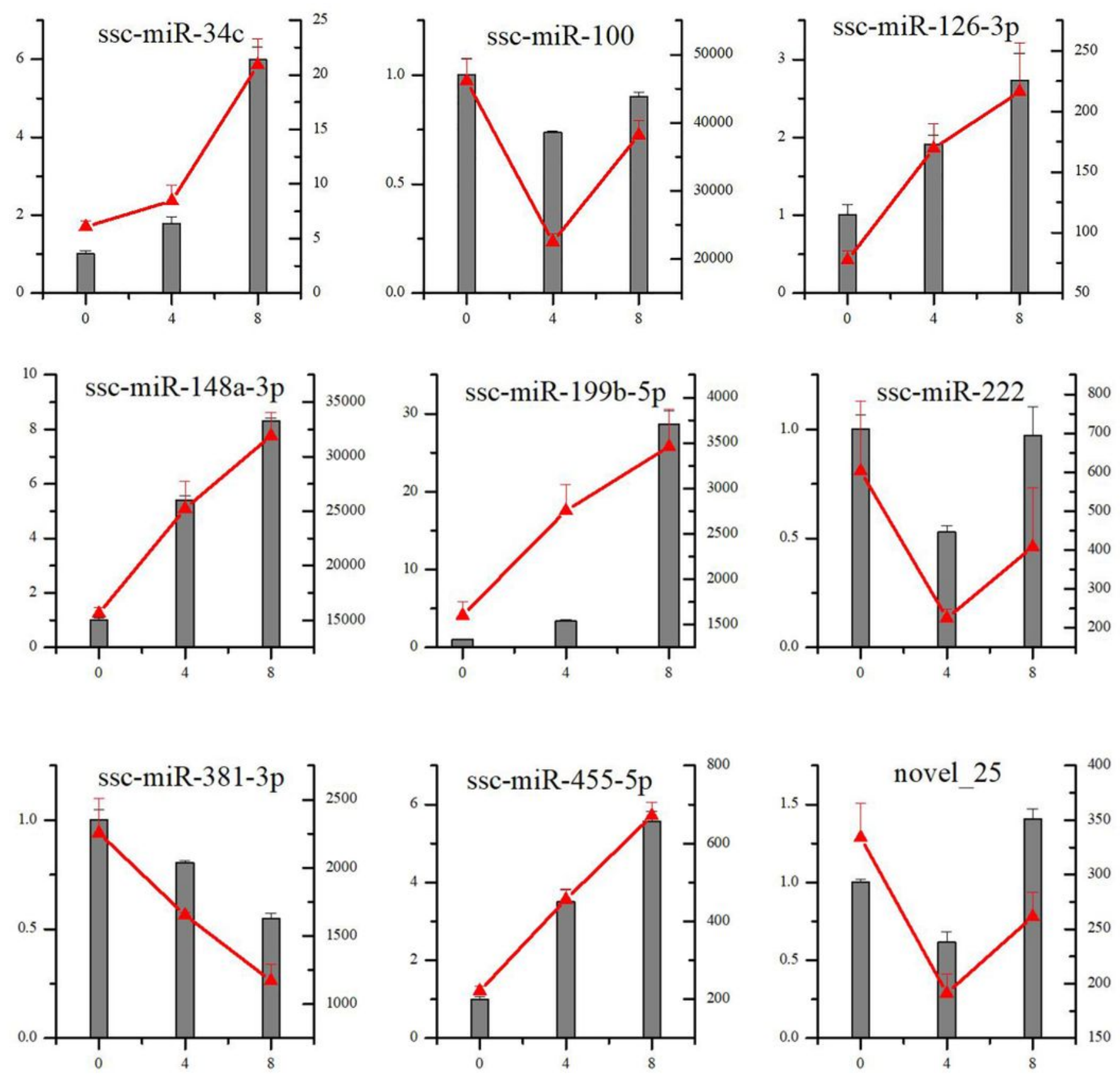

\section{Figure 6}

Validation of miRNAs involved in the three differentiation stages of lipid metabolism using qRT-PCR. Data from qRT-PCR are shown as column and Y-axis on the left, while the data from RNA-Seq are shown as line and $\mathrm{Y}$-axis on the right. 

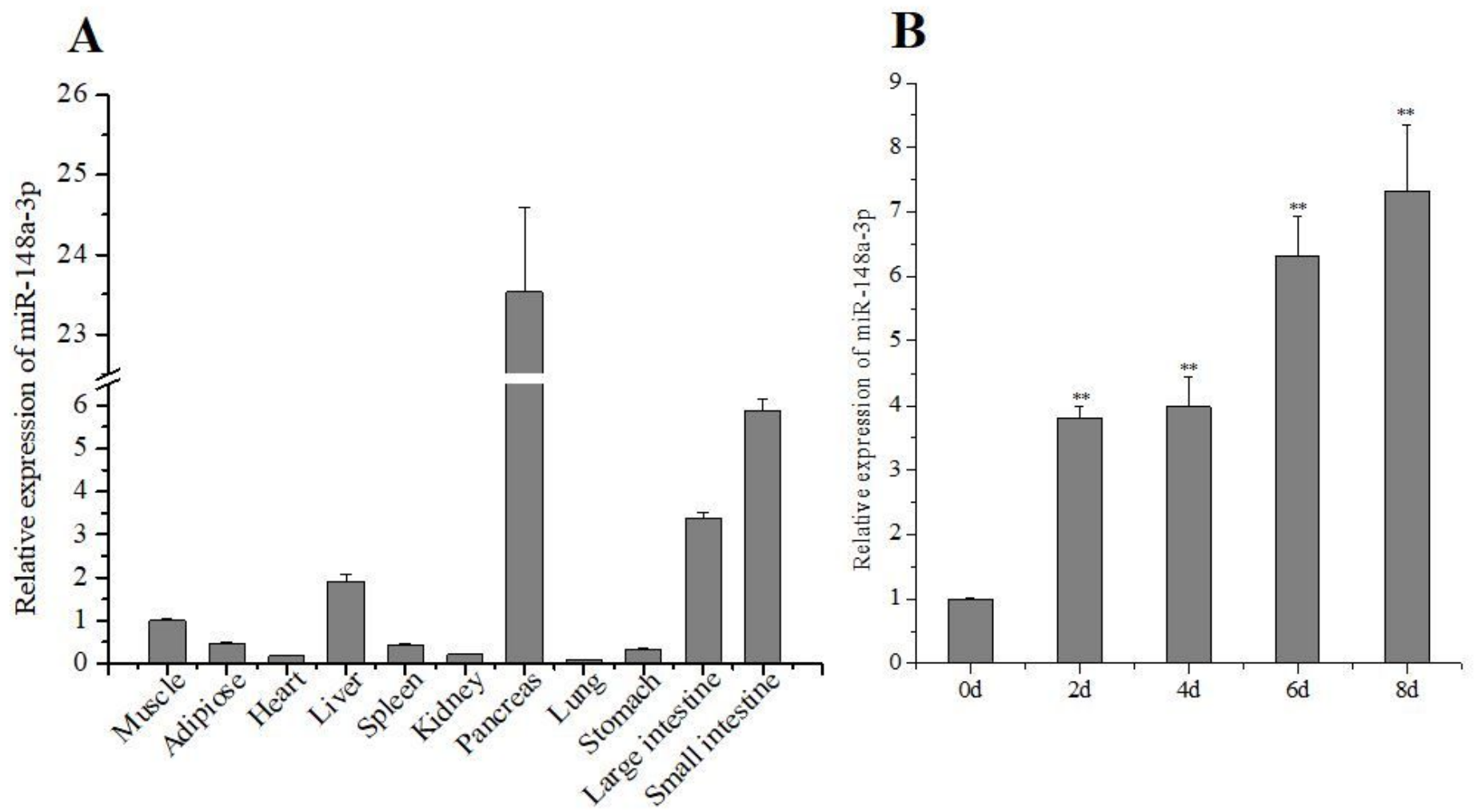

Figure 7

The relative expression level of miR-148a-3p in different tissues of China Guizhou Congjiang pigs $(A)$ and different differentiation stages of intramuscular preadipocyte (B). 
A
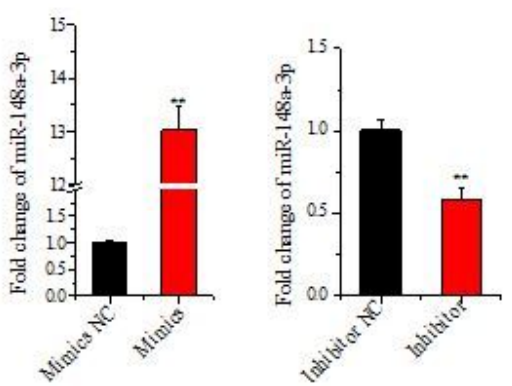

C
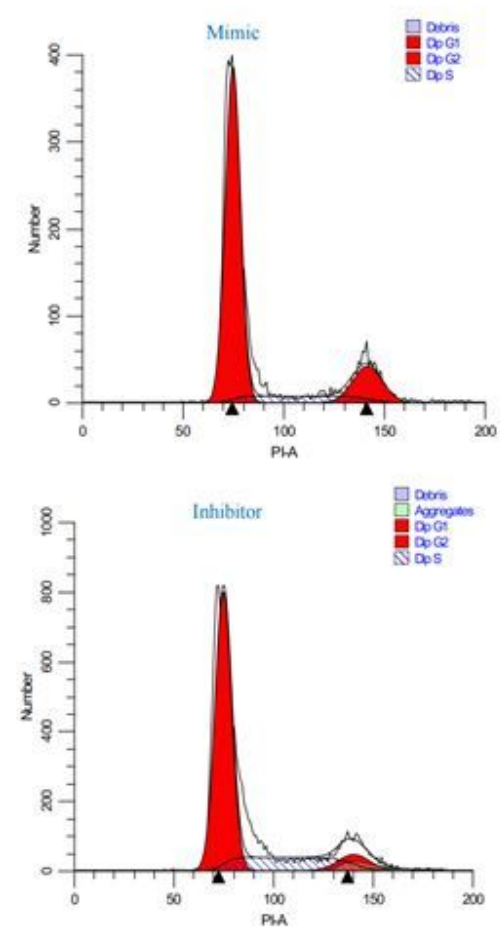

$\mathbf{E}$

Hoechst 33342

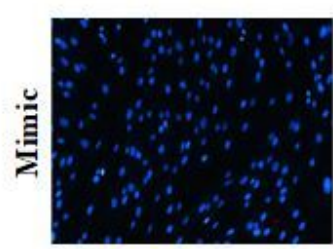

EdU
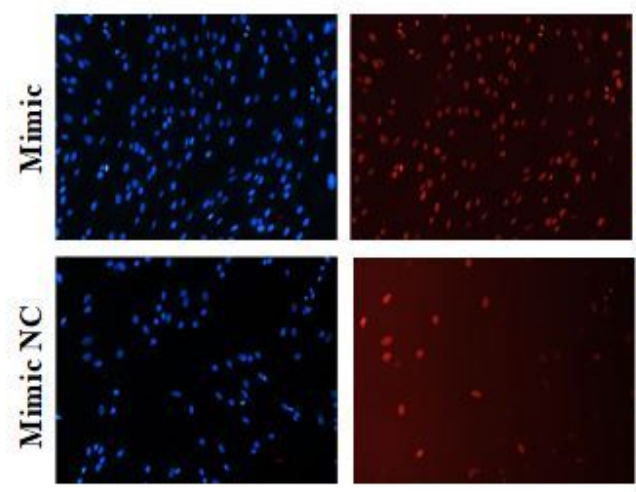

B
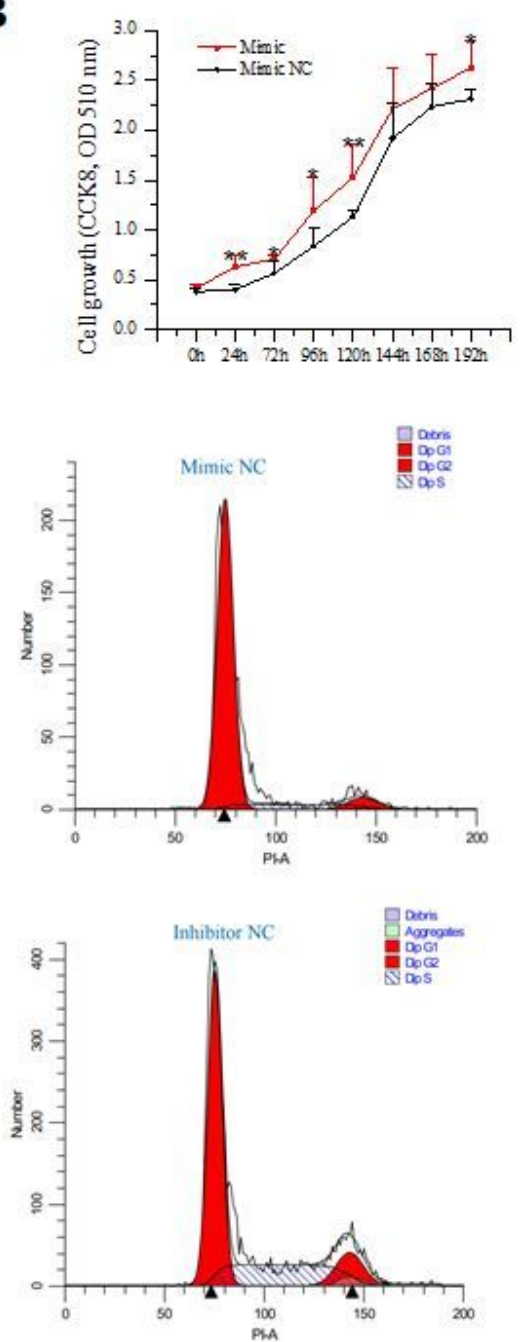

Merge

Hoechst 33342
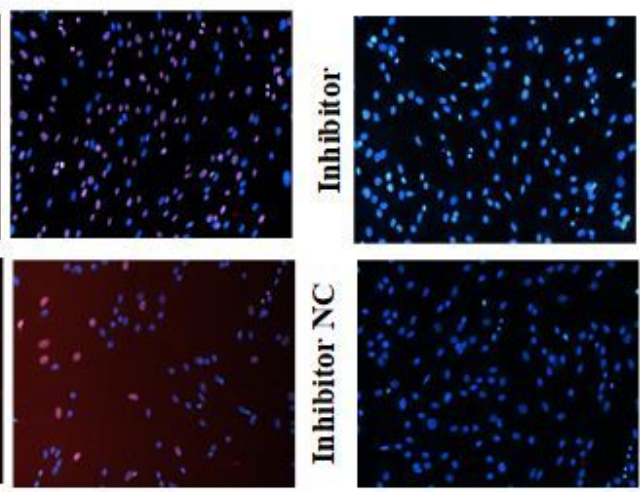

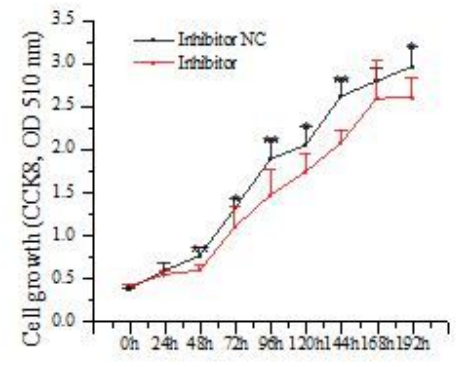

D
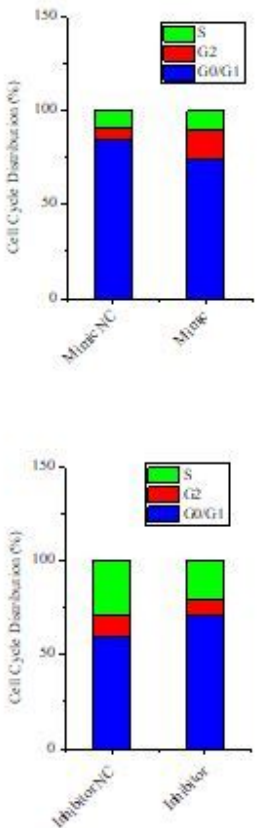

EdU
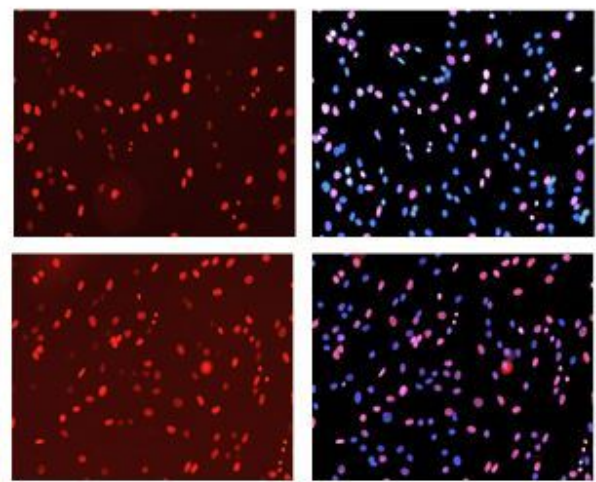

Figure 8

MiR-148a-3p promotes the proliferation of porcine intramuscular preadipocytes. (A) Relative expression of miR-148a-3p in porcine intramuscular preadipocyte transfected with miR-148a-3p mimics, mimics NC, inhibitor, and inhibitor NC. (B) Cell proliferation was detected using the cell counting kit 8(CCK-8). (C-D) Porcine intramuscular preadipocytes were transfected with miR-148a-3p mimics, mimics NC, inhibitor, inhibitor NC, and cell phases were analyzed using flow cytometry. (E) 5-ethynel-2'-deoxyuridine (EdU) was 
used to determine the proliferation. Data are shown as means \pm SEM for three individuals. ${ }^{*} \mathrm{P} \otimes 0.05,{ }^{\star *} \mathrm{P} \otimes$ 0.01 .

A

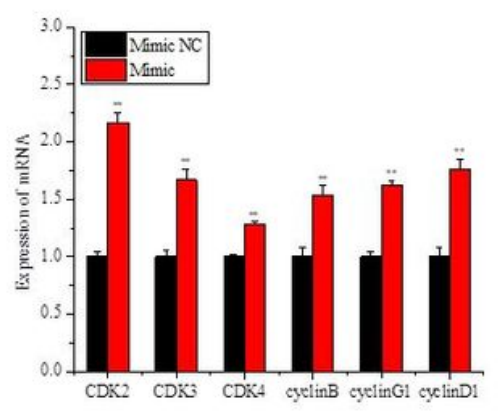

B

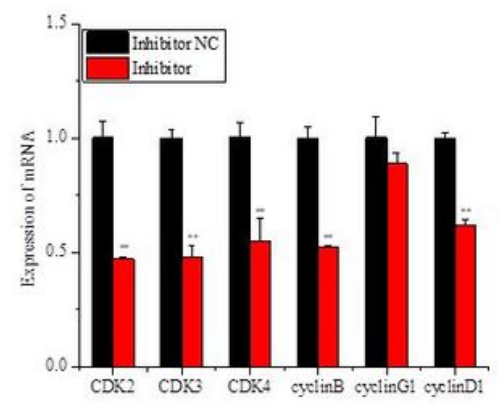

C
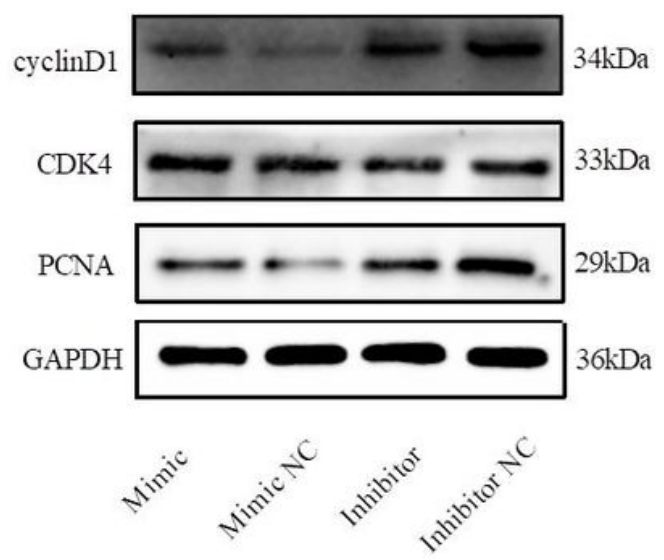

\section{Figure 9}

MiR-148a-3p promotes the proliferation of porcine intramuscular preadipocyte using qPCR and western bolt analysis. (A, B) The mRNA of proliferation marker genes (CDK2, CDK3, CDK4, cyclinB, cyclinG1, cyclinD1) was detected using real-time qPCR. (C) The protein level of cyclinD1, CDK4, and PCNA were detected by western blotting. Data are shown as means \pm SEM for three individuals. ${ }^{*} \mathrm{p} \llbracket 0.05,{ }^{\star \star} \mathrm{p} \otimes 0.01$. 
$\mathbf{A}$

B
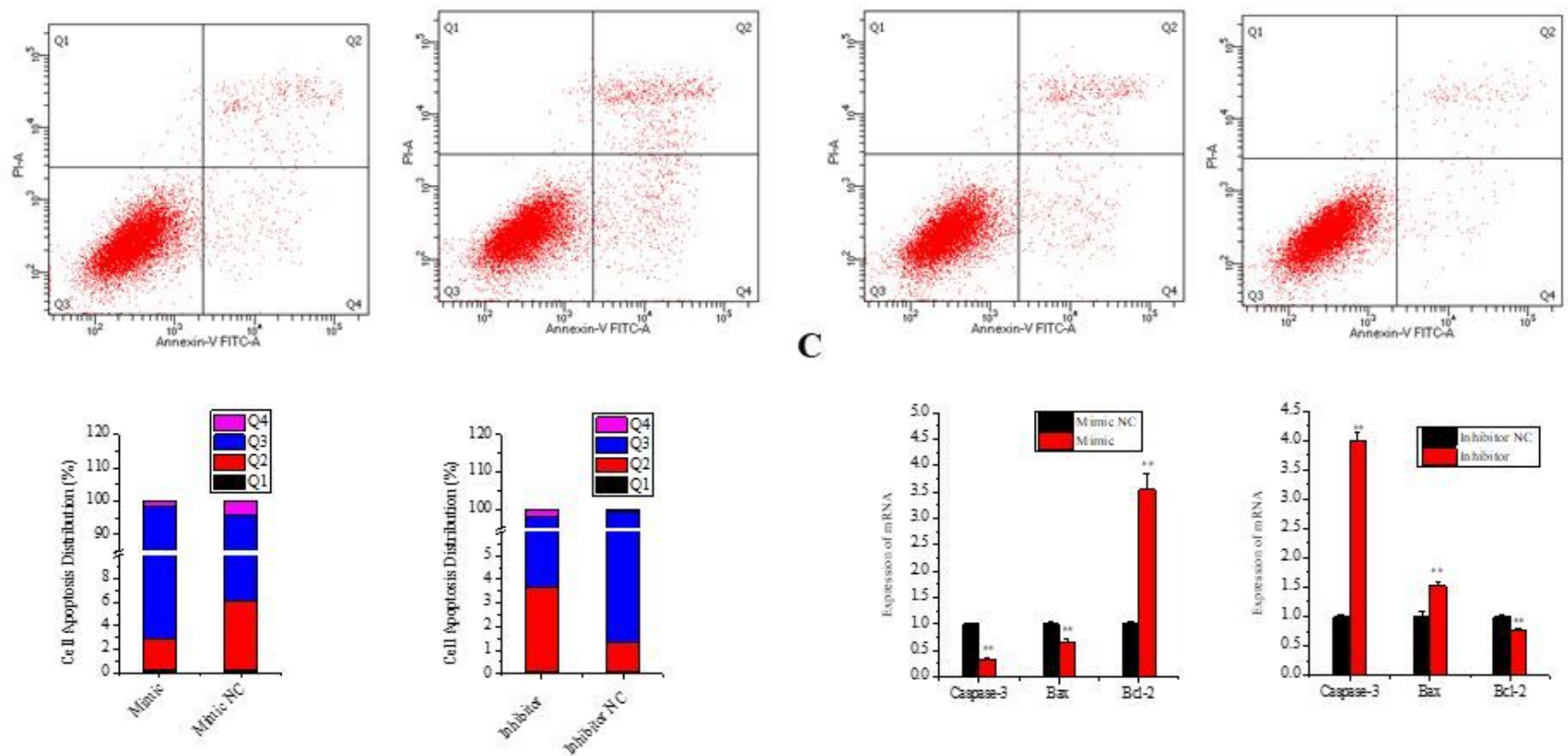

D

Hoechst 33342

PI
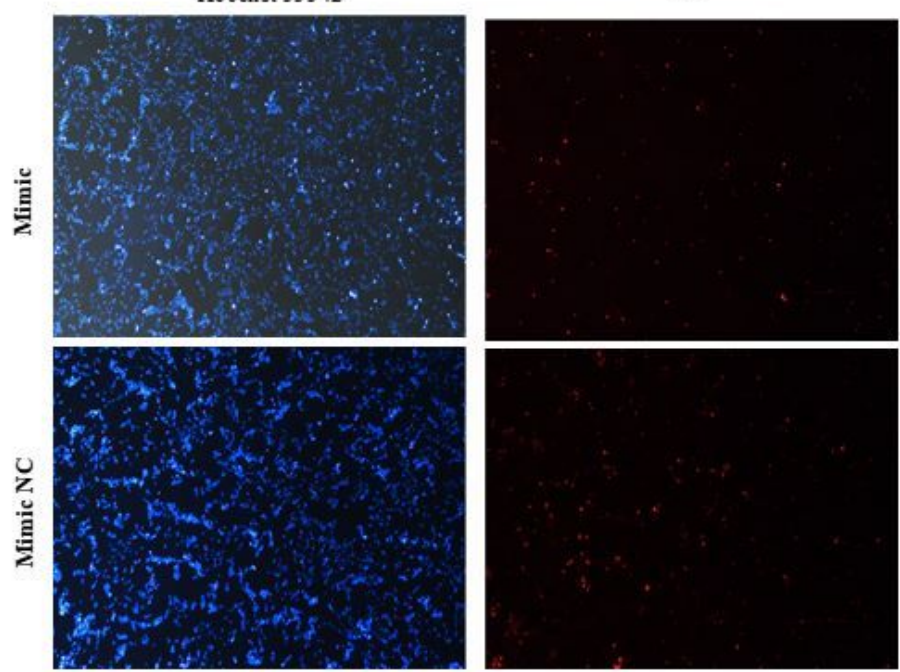
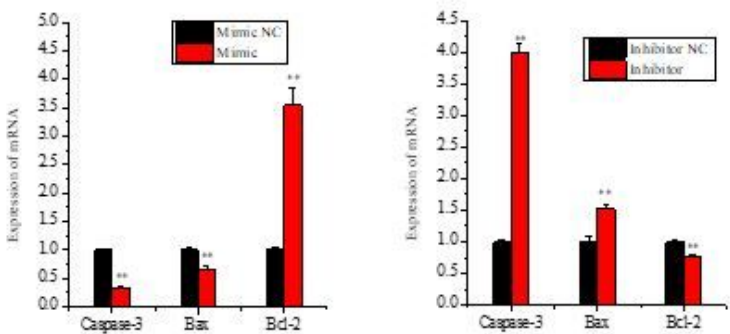

PI
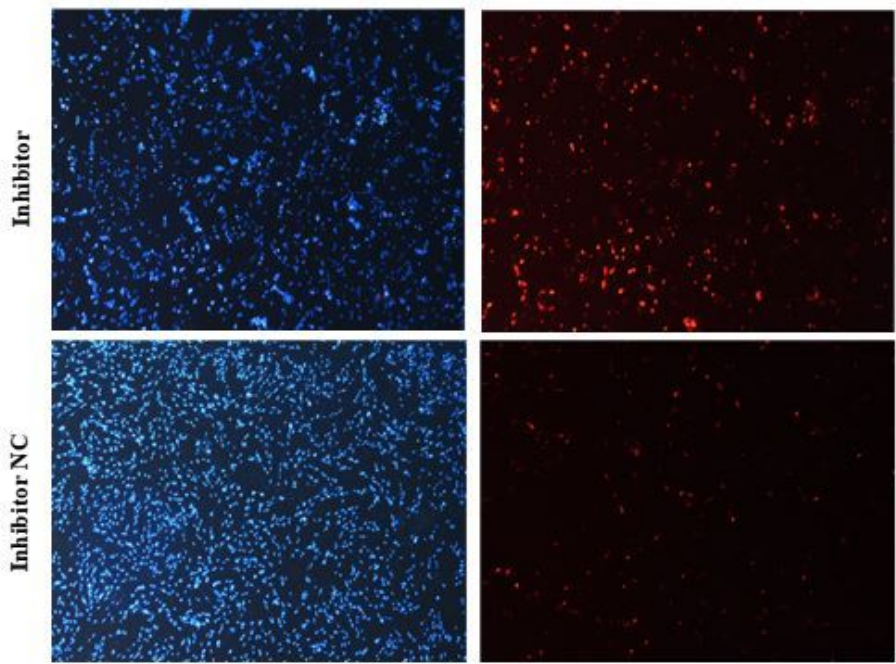

Figure 10

MiR-148a-3p inhibits apoptosis of porcine intramuscular preadipocyte. (A) Porcine intramuscular preadipocytes apoptosis was analyzed by Annexin V-FITC and PI binding using flow cytometry and counted (B). (C) The mRNA of apoptosis marker genes (Caspase-3, Bax, Bcl-2) was detected using realtime qPCR. (D) Cell apoptosis was detected by Hoechst 33342 and PI dual staining assays. Data are

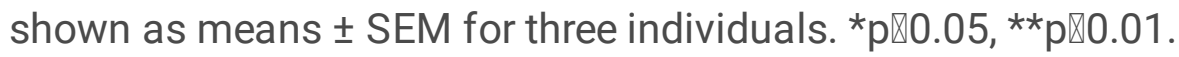


A

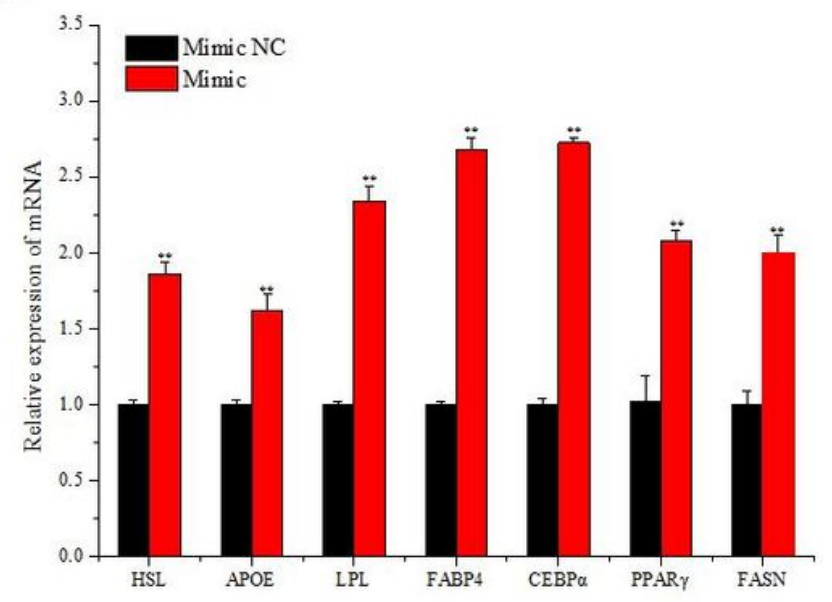

C

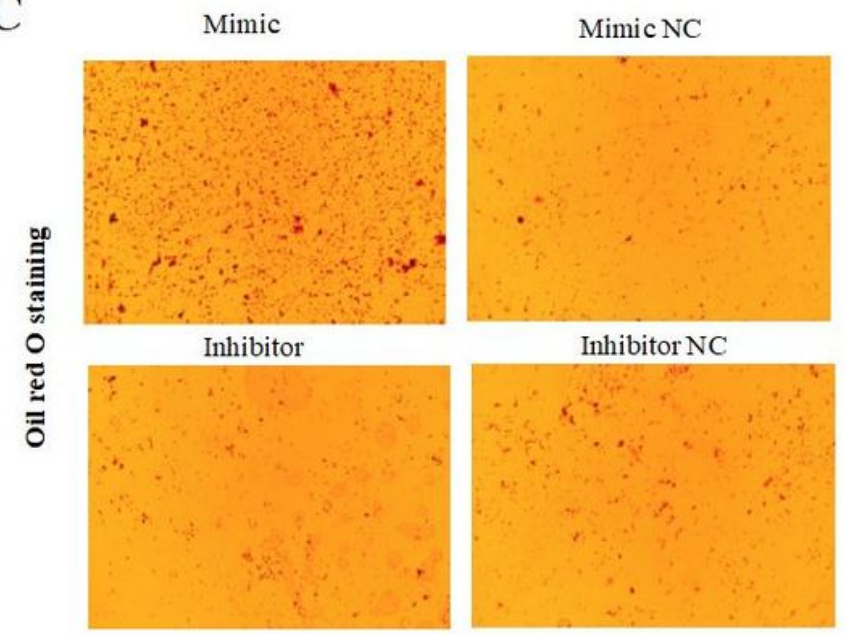

B

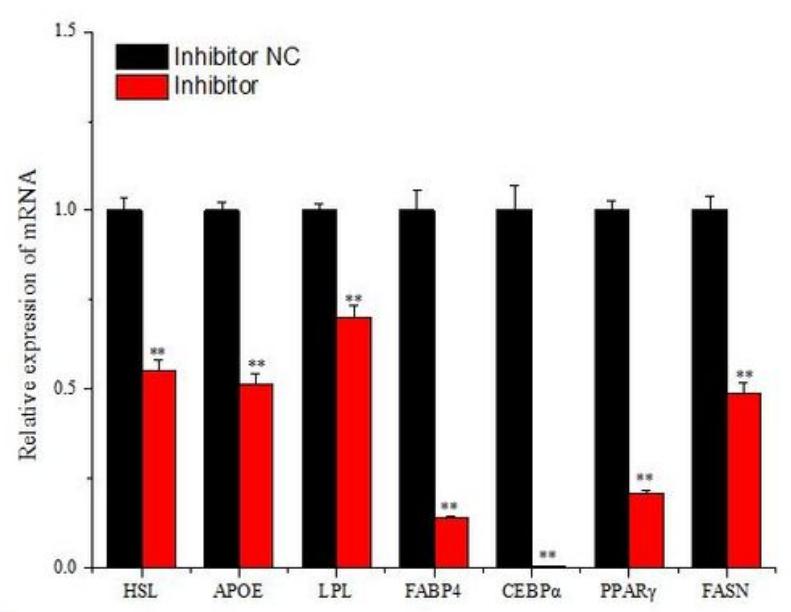

D

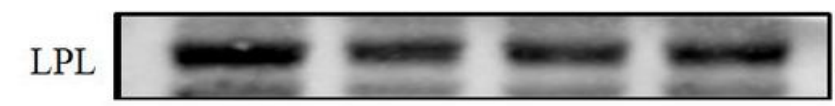

$53 \mathrm{kDa}$

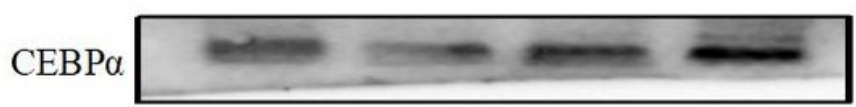

$38 \mathrm{kDa}$

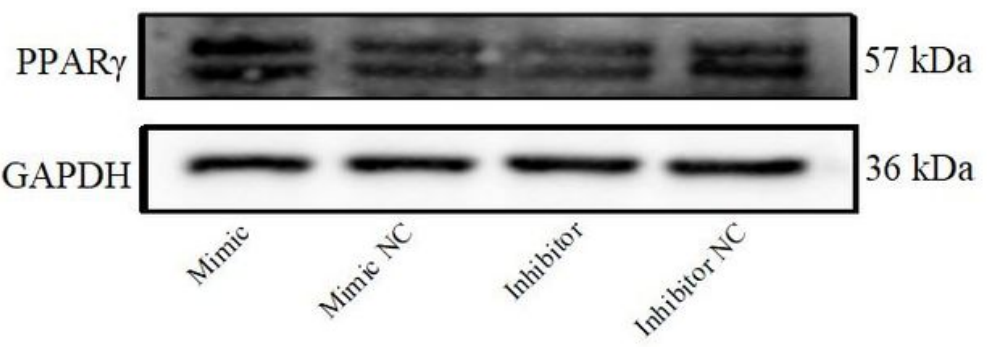

\section{Figure 11}

MiR-148a-3p promotes differentiation of porcine intramuscular preadipocyte. (A, B) The mRNA of differentiation marker genes were detected using real-time qPCR. (C) The lipid accumulation of porcine differentiated intramuscular adipocytes detected by Oil Red $O$ staining; (D) Protein levels of LPL, CEBPa, PPARy were examined by western blotting. All cells were measure at day 8 of adipogenic differentiation. Results were presented as means $\pm S E M, n=3 ; * p<0.05 ; * \star p<0.01$. 


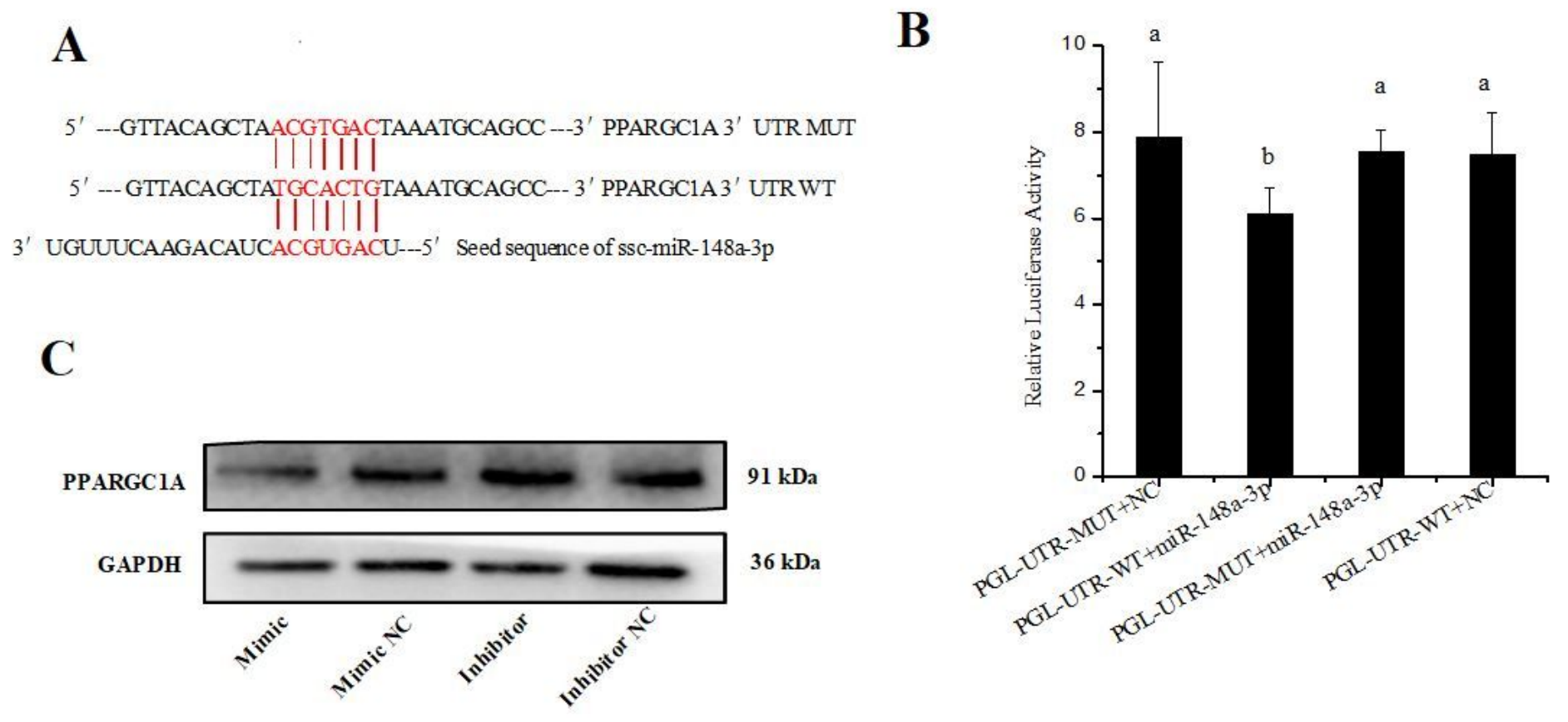

Figure 12

MiR-148a-3p promotes porcine intramuscular preadipocyte differentiation by targeting PPARGC1A. (A) RNAhybrid and TargetScan predicted miR-148a-3p binding sites in the PPARGC1A 3'UTR. (B) 293T cells were co-transfected with the miR-148a-3p mimics and PmirGLO-PPARGC1A-3'UTR-WT or PmirGLOPPARGC1A-3'UTR-WT, and Renilla and firefly luciferase activity was analyzed. (C) The protein expression of PPARGC1A was detected by western blotting. Different letters mean significant differences. 
A

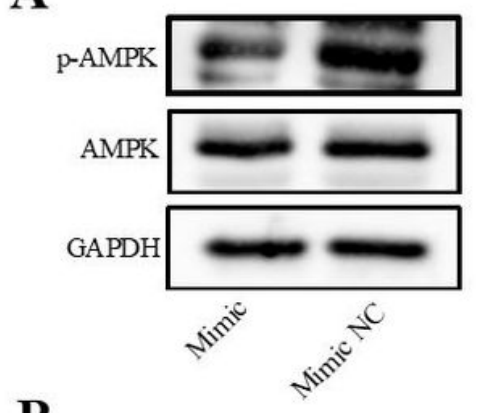

B

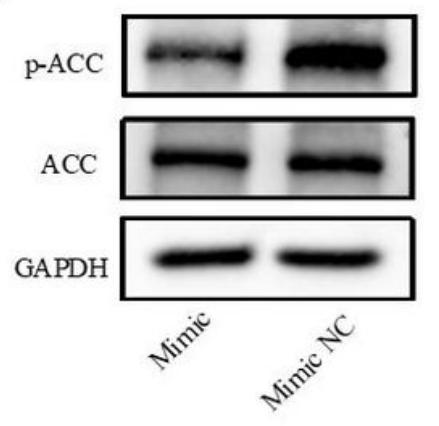

C

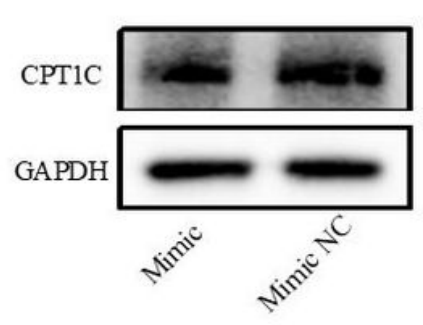

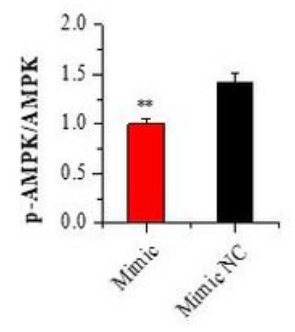

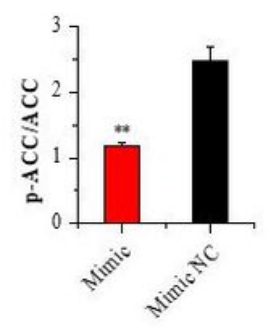

$\mathbf{F}$

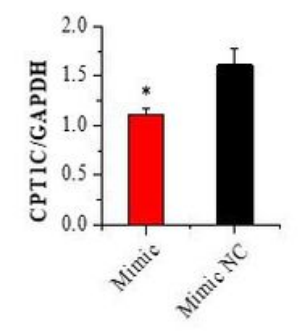

$\mathbf{E}$
D
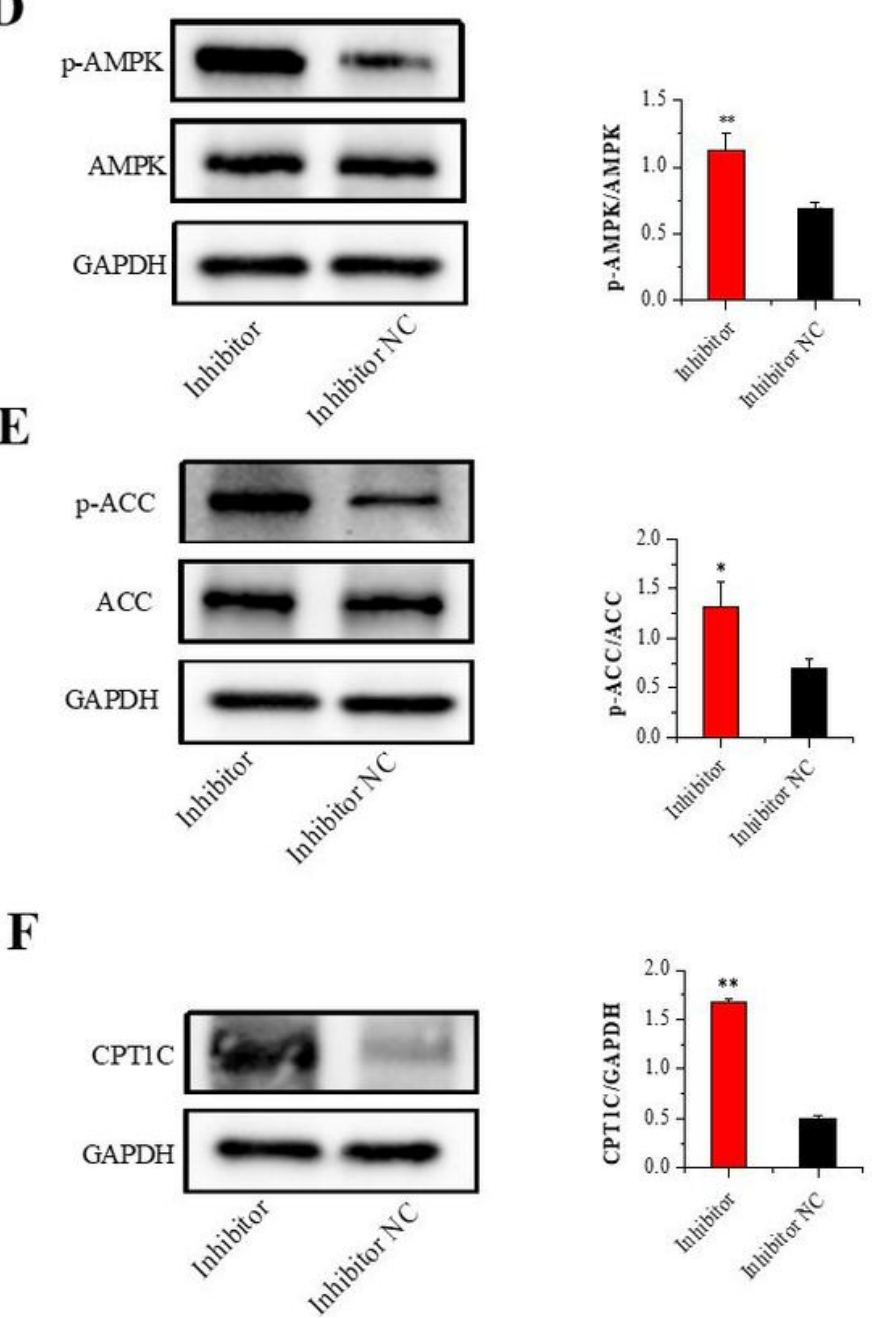

\section{Figure 13}

MiR-148a-3p promotes porcine intramuscular preadipocyte differentiation through inhibiting the AMPK/ACC/CPT-1 signaling pathway. (A, B) Protein levels of phosphorylated and total AMPK and ACC treated with miR-148a-3p mimic. (C) The protein level of CPT1C treated with miR-148a-3p mimic. (D, E) Protein levels of phosphorylated and total AMPK and ACC treated with miR-148a-3p inhibitor. (F) The protein level of CPT1C was treated with miR-148a-3p inhibitor. All cells were measure at day 8 of adipogenic differentiation. Results were presented as means $\pm S E M, n=3 ;{ }^{*} p<0.05 ;{ }^{*} p<0.01$.

\section{Supplementary Files}

This is a list of supplementary files associated with this preprint. Click to download.

- FigureS1.docx

- Figures2.docx

- Figures3.docx 
- Figures4.docx

- Figures5.docx

- FigureS6.docx

- Figures7.docx

- Figures8.docx

- TableS1.xIsx

- TableS10.xls

- TableS11.xls

- TableS12.xls

- Tables2.xIsx

- TableS3.doc

- Tables4.doc

- TableS5.doc

- TableS6.doc

- Tables7.xls

- Tables8.xls

- Tables9.xls 